\title{
EXPLORING THE ROLE OF HOST-TUMOR INTERACTIONS IN TUMOR GROWTH AND REGRESSION
}

\author{
BY \\ WILLIAM C. TROY (Department of Mathematics, University of Pittsburgh, Pittsburgh, \\ Pennsylvania 15260) \\ AND \\ STEWART J. ANDERSON (Department of Biostatistics, Graduate School of Public Health, \\ University of Pittsburgh, 312 Parran Hall, 130 DeSoto Street, Pittsburgh, Pennsylvania 15261)
}

\begin{abstract}
In the last 35 years the characterization of tumor growth using Gompertzian models has led to a new understanding of the spread of tumor cells, and has enabled researchers to develop novel therapeutic strategies to eradicate disease in some cases (see Ciron et al. (2003), Norton et al. (1976), Norton and Simon (1986), and Simon and Norton (2006)). A long standing assertion is that the Gompertzian framework does not allow characterizations of complex biological and clinical phenomena such as tumor regression and dormancy (see Retsky et al. (1998)). We propose a generalized Gompertzian system of delay-differential equations to study host-tumor interaction effects in the absence of external therapy. Our model is a parsimonious extension of the Norton et al. (1976) model: $N(t)$ denotes tumor volume, $G(t)$ represents host-tumor interactions, $N^{\prime}(t)=K_{1} N(t) G(t)$ and $G^{\prime}(t)=-K_{2} G(t-\tau)$, and $\tau \geq 0$ represents time of response of the host to the presence of tumor cells. The first step is to set $G(t)=\exp (\lambda t)$ and study $\lambda=K_{2} \exp (\lambda \tau)$. Setting $\lambda=\alpha(\tau)+\mathrm{i} \beta(\tau)$, we derive ODEs satisfied by $\alpha(\tau), \beta(\tau)$, and prove existence and qualitative properties of infinitely many branches of solutions. Therefore, $G(t)=\sum_{j \in I_{1}} c_{j} \exp \left(\alpha_{j}(\tau) t\right) \cos \left(\beta_{j}(\tau) t\right)+\sum_{k \in I_{2}} d_{k} \exp \left(\alpha_{k}(\tau) t\right) \sin \left(\beta_{k}(\tau) t\right)$, where $\exp \left(\alpha_{j}(\tau) t\right) \cos \left(\beta_{j}(\tau) t\right), \exp \left(\alpha_{k}(\tau) t\right) \sin \left(\beta_{k}(\tau) t\right)$ are eigenfunctions. Substituting $G(t)$ into the $N(t)$ ODE, we: (I) identify an "optimal immunological response" range $\tau>0$ where host-tumor interactions can cause a tumor to remain dormant, or regress from growth state into dormancy, and (II) replicate observed tumor growth in mammograms of 32 breast cancer patients.
\end{abstract}

1. Introduction. Although great strides have been made in the the last 35 years in the prevention and treatment of breast cancer, the American Cancer Society estimates that in 2013, 234,580 new cases of the disease will be diagnosed in the US which will

Received March 2, 2013.

2010 Mathematics Subject Classification. Primary 82B10.

E-mail address: troy@math.pitt.edu

E-mail address: sja@pitt.edu 
result in 40,930 deaths within a 5-year time period. Available information from the National Cancer Institute indicates that $61 \%$ of newly diagnosed breast cancers involve localized tumors. Only about $2.4 \%$ of patients with these localized tumors die within 5 years, but such tumors could potentially have uncontrollabe growth and/or metastasize over a period of a few years resulting in mortality later on. Hence, when confronted with the presence of a tumor (perhaps by mammographic detection), the clinician and patient need to answer several key questions: (i) will the tumor grow and evolve into invasive cancer, (ii) will it grow too slowly to ever become invasive, or (iii) will the tumor regress (presumably because of immune system effects) into a state of dormancy? There is emerging evidence that each of these scenarios can and does occur [36]. In this paper we propose a new model, a "generalized Gompertzian system", to investigate these issues. Our goal is to understand and predict how, in the absence of external treatment, internal host-tumor interactions (immune system) affect the evolution of tumor growth. To put our approach into perspective, below we give a description of the model (A), previous results ((B) and (C)), and our modeling and clinical goals (D).

(A) The Gompertz model. During the last 50 years the Gompertz equation has played an important role in modeling growth and treatment of tumors [3, 27, 28, 32, 35]. The Gompertz model [20] consists of

$$
\begin{aligned}
& \frac{d N(t)}{d t}=K_{1} N(t) G(t), N(0)=N_{0}>0, \\
& \frac{d G(t)}{d t}=-K_{2} G(t), G(0)=G_{1} \in \mathrm{R} .
\end{aligned}
$$

$N$ denotes tumor volume, $G$ is a feedback inhibitory variable, and $K_{1}>0, K_{2}>0$. Figure1(first row) shows growth and decay patterns exhibited by N and G. System (1.1) is equivalent to

$$
\frac{d N(t)}{d t}=K_{2} N(t) \ln \left(\frac{N_{\infty}}{N(t)}\right), N(0)=N_{0}>0,
$$

where $N_{\infty}=N_{0} \exp \left(\frac{K_{1} G_{1}}{K_{2}}\right)$. To see this, note that $G(t)=G_{1} \exp \left(-K_{2} t\right)$. Thus, (1.1) reduces to $\frac{d N(t)}{d t}=K_{1} N(t) G_{1} \exp \left(-K_{2} t\right), N(0)=N_{0}$. Integrate this equation from $t$ to $\infty$ to get (1.2).

(B) Tumor growth and treatment. In the 1960s Laird [27,28] used the Gompertz model to fit data of tumor growth. In 1976 Norton, Simon, Brereton and Bogden [32] discovered that solutions of (1.2) closely approximate two different types of tumor growth, namely, B-16 melanoma and 13276 rat carcinoma. For this they assumed that $G_{1}>0$ so that $N^{\prime}(t)>0 \forall t \geq 0$ and $N_{\infty}=N_{0} \exp \left(\frac{K_{1} G_{1}}{K_{2}}\right)>0$. An implicit assumption associated with the Gompertzian model is that large and small tumors are not as chemo-sensitive as intermediate sized tumors. To address this issue, Norton and Simon modified (1.2) to develop strategies for the optimal timing of the administration of a combination of drugs [33 35, 39]. Their modification is

$$
\frac{d N(t)}{d t}=K_{2} N(t) \ln \left(\frac{N_{\infty}}{N(t)}\right)(1-L(t)), N(0)=N_{0},
$$



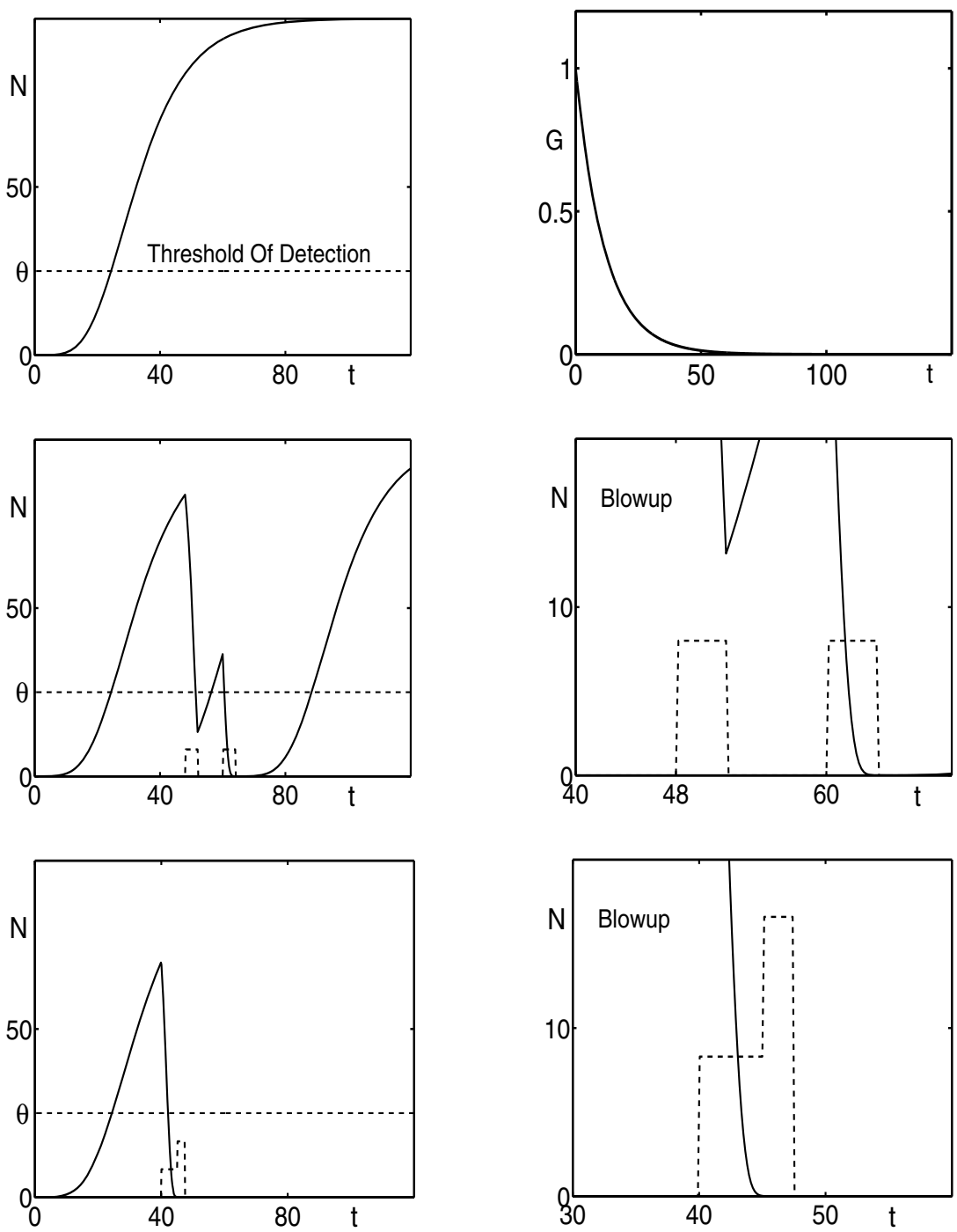

FIG. 1. Row 1. Graphs of $\mathrm{N}$ and $\mathrm{G}$ when $\left(K_{1}, K_{2}, N_{0}, G_{1}, N_{\infty}\right)=$ $(1, .087, .01,1,100)$ (Norton and Simon 33]). Cancer is clinically observed when $N(t)$ exceeds $\theta$, the threshold of detection. Row 2 . Standard levels of therapy (dashed curves) cause tumor volume $N(t)$ to regress below $\theta$. The tumor is not totally eradicated and it regrows. Row 3. The total level of therapy is the same as in Row 2 (i.e. the areas under $L(t)$ are the same), but now it increases significantly during the latter stage of its administration. This dose dense level of therapy causes $N(t)$ to decrease to zero at a first time $T^{*} \approx 47$. Subsequently, $N(t)=0$ for all $t \geq T^{*}$ and the tumor does not regrow. 
where $L(t)$ represents the effective level of therapy at time $t$. They found that if the level of therapy is significantly increased during the latter stage of its administration, then $N(t)$ can reach zero at a finite time $T^{*}>0$. Subsequently, $N(t)=0$ for all $t>T^{*}$. Thus, in contrast with strategies of using equal or diminishing intensity of agents, the strategy of "late intensification" of agents causes an improvement in tumor reduction (see Figure 1). This concept, later referred to as dose dense, was used as a driving force to generate a hypothesis for the development of a large, multi-phase III clinical trial (CALGB 9741). The results of that trial indicated that statistically significant improvements in overall survival and disease-free survival were observed in patients assigned to dose dense arms as compared to those assigned to the other arm of the same trial [2]. The original plan of late intensification was altered slightly in this trial. Nevertheless, the dose intensification strategy appears to optimize many pharmacokinetic properties of effective therapy while having only minor consequences with respect to toxicity compared to longer dosing schedules 40.

(C) Host-tumor interactions. Fisher [9, 16] demonstrated the fundamental role of host-tumor dynamics in a wide variety of tumors. His insights led to the implementation of two seminal clinical trials in the surgical management of breast cancer, namely NSABP Protocols B-04 [11,12] and B-06 [13, 14]. The results of these trials and others [26, 44, 45] reinforced one component of Fisher's hypotheses that "due to the systemic nature of the disease, variations in local or regional therapy are unlikely to have a substantial effect on overall survival" [9].

Tumor regression. The role of the immune system (host) in the spontaneous eradication of cancer cells has been explored for many years [16,43]. Early research focused on melanomas which are most commonly eradicated by the host 42 . While it was once thought to be rare that detectable carcinomas associated with solid tumors would spontaneously regress, recent findings by Zahl et al. [46] suggest that such regression is not uncommon. This may be a result of better detection methods which are now available so that smaller tumor burdens are observed and may be subsequently eradicated by the host 24, 46. In most cases, however, the host response is not sufficient to completely eradicate tumor cells and their subsequent proliferation. Thus, the complex mechanism of action by the host in its attempt to eradicate tumor cells is not well understood. This point is emphasized by McAllister et al. [30, who state "... many aspects of tumor biology can only be explained by a detailed understanding of both local and systemic interactions, yet we only have a fragmentary understanding of both processes."

Tumor dormancy. A fundamentally important phenomenon which involves complex interactions between host and tumor is tumor dormancy where tumor cells do not proliferate for a period of time (possibly many years) [31,37]. It has been hypothesized that the dormancy period itself may be a result of primary tumors secreting angiostatin into the bloodstream, thereby inhibiting blood vessel growth at sites typically associated with tumor metastases [30. However, such cells may leave the dormancy state and begin to proliferate at a high rate $([10,25,31])$. Many mechanisms for leaving a tumor dormancy state have been proposed, including: 
(i) Angiogenesis (neovascularization) [18: the identification of an angiogenic diffusible factor derived from tumors was made initially by Greenblatt and Shubik in 1968 [21].

(ii) Persistent circulating tumor cells may metastasize at a point in time when the host system is perturbed [31].

A long-standing belief is that Gompertzian kinetics cannot exhibit such complicated behavior as tumor dormancy or regression [3, 41. One of our primary goals (see part (D) is to show that this is false, and that an extension of the original Gompertz model to a generalized Gompertzian system does indeed exhibit both regression and dormancy behavior.

(D) Goals. The Norton and Simon 1976-1977 studies of tumor growth [33, 34, allow only a limited number of host-tumor interactions. In [34,35] they introduced the external treatment term $L(t)$ (dashed curves in Figure 1) into (1.3) to study how an external signal (treatment) affects tumor growth. In this paper our goal is to understand how host-tumor interactions affect tumor growth, tumor regression, and tumor dormancy in the absence of external therapy. For this we extend (1.1) and derive a generalized Gompertzian model. Our approach is to introduce a delay, $\tau \geq 0$, which we interpret to represent the time of response of the host to the presence of the tumor, into the inhibitory feedback variable $G$, and study the behavior of solutions of

$$
\begin{aligned}
& \frac{d N(t)}{d t}=K_{1} N(t) G(t), \\
& \frac{d G(t)}{d t}=-K_{2} G(t-\tau) .
\end{aligned}
$$

The same interpretation of $\tau$ was given by Glass and Mackey [19] in the study of forms of leukemia where the rates of stem cell proliferation entail a time delay. Our investigation of the generalized Gompertzian model (1.4) has the following theoretical and clinical goals:

Goal I. We will demonstrate the practical utility of (1.4) by showing how host-tumor interactions affect "transient" large time tumor growth behavior, and by identifying an "optimal immunological response" range of positive $\tau$ where host-tumor interactions cause a tumor to remain in a dormant state, or regress from a growth state into a dormant state.

Goal II. We show how to use (1.4) and results of Goal I to: (i) accurately replicate data from mammograms of 32 breast cancer patients, and (ii) predict future growth patterns for each data set.

The first step in achieving Goals I and II is to understand the behavior of

$$
\frac{d G(t)}{d t}=-K_{2} G(t-\tau), t \geq 0, \tau \geq 0 .
$$

A typical initial condition for (1.5) is $G t)=g(t),-\tau \leq t \leq 0$, where $g(t)$ is a given function. For such initial conditions, a simple method to solve (1.5) is to integrate, interval by interval of length $\tau$. However, this continuation type process does not usually result in useful formulas for understanding behavior of solutions, especially for large $t$ values ([1, p. 53). A second approach is to construct an eigenvalue/eigenvector expansion of solutions ([1], p. 55). For this, substitute $G(t)=e^{\lambda t}$ into (1.5), and obtain

$$
\lambda=-K_{2} e^{-\lambda \tau} \text {. }
$$


Let $\lambda=\alpha+i \beta$. Then (1.6) becomes $\alpha+i \beta=-K_{2} e^{-(\alpha+i \beta) \tau}$. Separating real and imaginary parts, we obtain the nonlinear algebraic system

$$
\alpha+K_{2} e^{-\alpha \tau} \cos (\beta \tau)=0, \beta-K_{2} e^{-\alpha \tau} \sin (\beta \tau)=0 .
$$

Analysis of (1.6)-(1.7). In order to construct and understand the full impact of eigenvalue/eigenvector expansion solutions of the delay equation (1.5) on tumor volume, we must first determine the behavior, in terms of $\tau$, of each individual branch of solutions of the eigenvalue equations (1.6) and (1.7). For this it is necessary to answer the following fundamental questions:

$\left(\mathbf{Q}_{1}\right)$ When $\tau>0$, how many branches of solutions does (1.6)-(1.7) have?

$\left(\mathbf{Q}_{2}\right)$ For a given branch, let $\left(\tau_{\min }, \tau_{\text {min }}\right)$ denote its maximal positive interval of existence. Along each branch, is $\lambda(\tau)=\alpha(\tau)+\mathrm{i} \beta(\tau)$ a $\mathrm{C}^{1}$ function of $\tau \in\left(\tau_{\min }, \tau_{\max }\right)$ ?

$\left(\mathbf{Q}_{3}\right)$ Along each branch we associate the key subsets

$$
\mathrm{U}=\left\{\tau \in\left(\tau_{\min }, \tau_{\max }\right) \mid \beta(\tau)=0\right\} \text { and } \mathrm{W}=\left\{\tau \in\left(\tau_{\min }, \tau_{\max }\right) \mid \beta(\tau) \neq 0\right\} .
$$

Note that $\lambda$ is real if $\tau \in \mathrm{U}$, and complex if $\tau \in \mathrm{W}$. Along each branch, what are the subsets of $\mathrm{U}$ and $\mathrm{W}$ where $\alpha(\tau)<0, \alpha(\tau)=0, \alpha(\tau)>0$ ?

$\left(\mathbf{Q}_{4}\right)$ Along each branch, what are the limiting values

$$
\lim _{\tau \rightarrow \tau_{\min }^{+}}(\alpha(\tau), \beta(\tau)) \text { and } \lim _{\tau \rightarrow \tau_{\max }^{-}}(\alpha(\tau), \beta(\tau)) ?
$$

Previous results. The classic text by Bellman and Cooke [1] devotes much attention to analyzing equations similar to (1.6). There (Chs. 4 and 12), the focus is on using Laplace transform and contour integration techniques to derive asymptotic estimates for the location of eigenvalues when $|\lambda| \gg 1$. These methods give partial answers to $\left(\mathbf{Q}_{1}\right)$ only when $|\lambda| \gg 1$. They do not apply when $|\lambda|$ is not large, nor do they give insight to resolve the issues raised in $\left(\mathbf{Q}_{2}\right)-\left(\mathbf{Q}_{4}\right)$. Another classic text, by Diekman et al. [6], uses functional analysis, complex analysis and nonlinear methods (e.g. semigroup theory, spectral methods, contour integration, etc.) to analyze (1.5) and (1.6). These methods give only partial answers to $\left(\mathbf{Q}_{1}\right)-\left(\mathbf{Q}_{3}\right)$, and do not provide useful techniques to answer $\left(\mathbf{Q}_{4}\right)$. Jeffrey et al. [23] use a winding number method to study existence of branches of solutions of (1.6). Their method also gives only partial answers to $\left(\mathbf{Q}_{1}\right)$, and does not apply to $\left(\mathbf{Q}_{2}\right)-\left(\mathbf{Q}_{4}\right)$.

Our approach to analyzing (1.6)-(1.7). We develop a new ODE based technique which allows us to answer $\left(\mathbf{Q}_{1}\right)-\left(\mathbf{Q}_{4}\right)$. In particular, we derive and analyze differential equations satisfied by $\alpha(\tau)$ and $\beta(\tau)$. For this, differentiate $\alpha$ and $\beta$ in (1.7) with respect to $\tau$ and obtain

$$
\begin{aligned}
& \alpha^{\prime}=\frac{1}{(1+\alpha \tau)^{2}+(\beta \tau)^{2}}\left(\beta^{2}-\alpha^{2}-\alpha \beta^{2} \tau-\alpha^{3} \tau\right), \\
& \beta^{\prime}=-\frac{\beta}{(1+\alpha \tau)^{2}+(\beta \tau)^{2}}\left(2 \alpha+\alpha^{2} \tau+\beta^{2} \tau\right) .
\end{aligned}
$$

Imaginary eigenvalues found in Section 3 provide the initial values

$$
\alpha\left(\frac{(4 j+1) \pi}{2 K_{2}}\right)=0 \text { and } \beta\left(\frac{(4 j+1) \pi}{2 K_{2}}\right)=K_{2}, j \geq 0 .
$$

Theorem 3.1 (see Section 4) contains our main theoretical advance. There we answer $\left(\mathbf{Q}_{1}\right)-\left(\mathbf{Q}_{4}\right)$ by proving the existence and global properties of infinitely many branches of 
$\mathrm{C}^{1}$ solutions of (1.10)-(1.11) Our ODE approach is completely new and independent of previous techniques $([1,6,23])$ used to analyze system (1.7). The analysis of (1.10)-(1.11) provides a formidable challenge since (1.10) is a nonautonomous system, hence, standard techniques such as phase plane analysis do not apply. Our ODE approach to proving Theorem 3.1 provides the first global analysis of (1.7), and leads to the following distinct advances over previous studies:

(i) We prove the interval of existence of the branches are not all the same.

(ii) We resolve the issues raised in $\left(\mathbf{Q}_{1}\right)-\left(\mathbf{Q}_{4}\right)$ for each branch.

(iii) Our approach is completely constructive: for each $j \geq 0$ we can solve (1.10)-(1.11) numerically, and compute $\alpha_{j}(\tau)$ and $\beta_{j}(\tau)$ over the entire interval of existence (Figures 4 and 5 in Section 4).

Goals I and II. We show how to use Theorem 3.1 to construct eigenvalue expansions which allow us to address the modeling and clinical data issues stated above in Goals I and II. It follows from Theorem 3.1 that

$$
G=\sum_{j \in I_{1}} c_{j} \exp \left(\alpha_{j}(\tau) t\right) \cos \left(\beta_{j}(\tau) t\right)+\sum_{k \in I_{2}} d_{k} \exp \left(\alpha_{k}(\tau) t\right) \sin \left(\beta_{k}(\tau) t\right)
$$

where $\exp \left(\alpha_{j}(\tau) t\right) \cos \left(\beta_{j}(\tau) t\right)$ and $\exp \left(\alpha_{k}(\tau) t\right) \sin \left(\beta_{k}(\tau) t\right)$ are eigenfunctions corresponding to eigenvalues $\lambda=\alpha(\tau)+i \beta(\tau)$, and coefficients $c_{j}$ and $d_{k}$ determine the strength of contribution of the eigenfunctions. Substituting (1.12) into (1.4) transforms the tumor volume equation into

$$
\frac{d N}{d t}=K_{1} N\left(\sum_{j \in I_{1}} c_{j} \exp \left(\alpha_{j}(\tau) t\right) \cos \left(\beta_{j}(\tau) t\right)+\sum_{k \in I_{2}} d_{k} \exp \left(\alpha_{k}(\tau) t\right) \sin \left(\beta_{k}(\tau) t\right)\right)
$$

In Section 5 we show how to use (1.13) to resolve all of the tumor-host interaction issues in Goal I. In Section 6 we attain Goal II by demonstrating how to apply (1.13) to accurately replicate the data sets for tumors detected in mammogram examinations of 32 breast cancer patients. Finally, in Section 7 we make conclusions and give suggestions for future research.

2. Real and imaginary eigenvalues. Real eigenvalues. In this section we prove the existence and qualitative properties of a unique branch of solutions of (1.7) for which $\lambda$ is real. Solutions along this branch form the leading terms in eigenfunction expansion (4.1) in Section 5. Thus, although the results for the real eigenvalue case are well known, it is necessary to include the details to obtain the most complete understanding possbile of global behavior of solutions of (1.7). Let $\beta=0$ so that $\lambda=\alpha+i \beta=\alpha$ is real. Then (1.7) reduces to

$$
\alpha+K_{2} e^{-\alpha \tau}=0, K_{2}>0 \text {. }
$$

If $\lambda=\alpha \geq 0$, then (2.1) has no solution. When $\lambda=\alpha<0$ we solve (2.1) for $\tau$ and obtain

$$
\tau=-\frac{1}{\alpha} \ln \left(\frac{-\alpha}{K_{2}}\right),-\infty<\alpha<0 .
$$



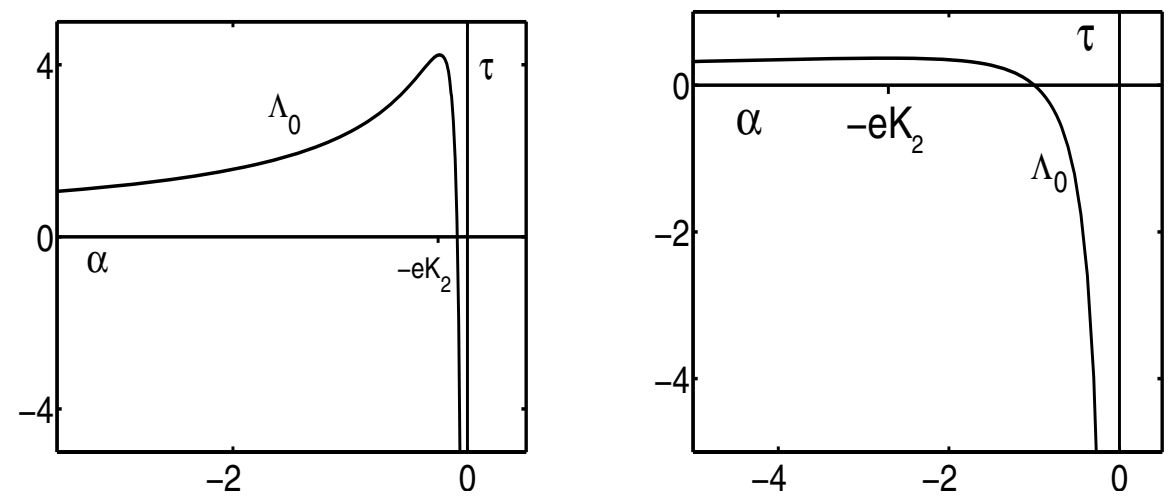

FIG. 2. The graph of curve $\Lambda_{0}$ defined by $\tau(\alpha)=-\frac{1}{\alpha} \ln \left(\frac{-\alpha}{K_{2}}\right)$ when $\left(\beta, K_{2}\right)=(0, .087)$ (left panel) and $\left(\beta, K_{2}\right)=(0,1)$ (right panel). For each $\tau \in\left(0, \tau^{*}\right)=\left(0, \frac{1}{e K_{2}}\right)$ there are two values, $\alpha=r_{1}(\tau)$ and $\alpha=r_{2}(\tau)$ such that $\left(\tau, r_{i}(\tau)\right) \in \Lambda_{0}$, and $r_{2}(\tau)<<r_{1}(\tau)$. Along $\Lambda_{0}$ the absolute maximum occurs at $\left(\alpha^{*}, \tau^{*}\right)=\left(-e K_{2}, \frac{1}{e K_{2}}\right)$. Thus, real eigenvalues do not exist when $\tau>\tau^{*}$.

Properties. (i) The function $\tau(\alpha)=-\frac{1}{\alpha} \ln \left(\frac{-\alpha}{K_{2}}\right)$ is graphed in Figure 2 2 It has a unique relative maximum value $\tau^{*}=\frac{1}{e K_{2}}$ at $\alpha=-e K_{2}$, no relative minimum, a unique zero at $\alpha=-K_{2}$, and

$$
\lim _{\alpha \rightarrow-\infty} \tau(\alpha)=0 \text { and } \lim _{\alpha \rightarrow 0^{-}} \tau(\alpha)=-\infty .
$$

The corresponding branch $\Lambda_{0}$ (see Figure 2) of real eigenvalue solutions of (1.7) is defined by

$$
\Lambda_{0}=\left\{(\alpha, \beta, \tau) \mid \alpha<0, \beta=0, \tau=-\frac{1}{\alpha} \ln \left(\frac{-\alpha}{K_{2}}\right)\right\}
$$

(ii) For each $\alpha \in(-\infty, 0)$ the point $(\alpha, 0, \tau)=\left(\alpha, 0,-\frac{1}{\alpha} \ln \left(\frac{-\alpha}{K_{2}}\right)\right) \in \Lambda_{0}$, and for each $c \in \mathrm{R}$ the function $G=c e^{\alpha t}$ solves the corresponding delay equation

$$
\frac{d}{d t} G(t)=-K_{2} G\left(t+\frac{1}{\alpha} \ln \left(\frac{-\alpha}{K_{2}}\right)\right) .
$$

(iii) There are no real eigenvalues when $\tau>\tau^{*}=\frac{1}{e K_{2}}$ where $\alpha=-e K$. For each $\tau \in\left(0, \tau^{*}\right)=\left(0, \frac{1}{e K_{2}}\right)$ there are exactly two negative $\alpha$ values, $r_{2}<r_{1}<0$, such that $\left(r_{1}, 0, \tau\right) \in \Lambda_{0}$ and $\left(r_{2}, 0, \tau\right) \in \Lambda_{0}$. Corresponding to $r_{1}$ and $r_{2}$ is a two-parameter family

$$
G=G_{1} e^{r_{1} t}+G_{2} e^{r_{2} t}
$$

Imaginary eigenvalues. We show that there are infinitely many solutions of (1.7) for which $\lambda$ is purely imaginary, and that the corresponding solutions of the delay equation (1.5) are periodic. In Section 3 we show how each periodic solution can be continued to a global branch of solutions. Let $\alpha=0$ so that $\lambda=i \beta$ is purely imaginary. Then (1.7) 

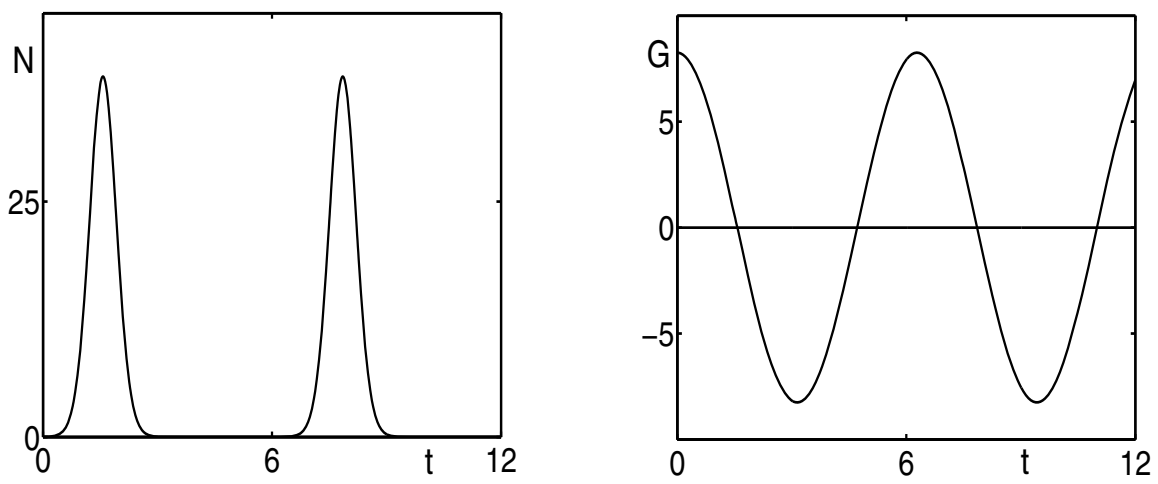

FIG. 3. Graphs of $N$ and $G$ components of a periodic solution of (1.4) corresponding to $(\alpha, \beta, \tau)=\left(0,1, \frac{\pi}{2}\right)$ in the first equation of 2.9). Here $\left(N(0), K_{1}, K_{2}, j\right)=(.01,1,1,0)$ so that $\tau=\tau_{0}=\frac{\pi}{2}$, hence $G(t)=8.5 \cos (t)$ is the corresponding periodic solution of $G^{\prime}(t)=$ $-G\left(t-\frac{\pi}{2}\right)$.

reduces to

$$
\cos (\beta \tau)=0, \beta-K_{2} \sin (\beta \tau)=0 .
$$

Four families of solutions of (2.7) are given by

$$
(\beta, \tau)=\left\{\begin{array}{l}
\left( \pm K_{2}, \frac{(4 j+1) \pi}{2 K_{2}}\right), j=0,1,2, \ldots \\
\left( \pm K_{2},-\frac{(2 j+1) \pi}{2 K_{2}}\right), j=1,3,5 \ldots
\end{array}\right.
$$

For values defined in (2.8), the corresponding solutions of (1.7) are

$$
(\alpha, \beta, \tau)=\left\{\begin{array}{l}
\left(0, \pm K_{2}, \frac{(4 j+1) \pi}{2 K_{2}}\right), j=0,1,2, \ldots \\
\left(0, \pm K_{2},-\frac{(2 j+1) \pi}{2 K_{2}}\right), j=1,3,5 \ldots
\end{array}\right.
$$

Thus, for each nonnegative integer $j$, the points $(\alpha, \beta, \tau)=\left(0, \pm K_{2}, \frac{(4 j+1) \pi}{2 K_{2}}\right)$ generate the two-parameter family $G(t)=c_{1} \sin \left(K_{2} t\right)+c_{2} \cos \left(K_{2} t\right)$ of periodic solutions of the delay equation

$$
\frac{d}{d t} G(t)=-K_{2} G\left(t-\frac{(4 j+1) \pi}{2 K_{2}}\right) .
$$

Figure 3 illustrates a periodic solution of (1.4) when $K_{1}=K_{2}=1, j=0$ and $\tau=\tau_{0}=\frac{\pi}{2}$. Similarly, for each integer $j \in\{1,3,5, \ldots\}$, the points $(\alpha, \beta, \tau)=\left(0, \pm K_{2},-\frac{(2 j+2) \pi}{2 K_{2}}\right)$ generate periodic solutions $G(t)=d_{1} \sin \left(K_{2} t\right)+d_{2} \cos \left(K_{2} t\right)$ of delay equation $\frac{d}{d t} G(t)=$ $-K_{2} G\left(t+\frac{(2 j+1) \pi}{2 K_{2}}\right)$.

3. Complex eigenvalues. In this section we prove, in Theorem 3.1, the existence of infinitely many branches of complex eignevalue solutions of (1.7). The first step of the 
proof is to assume that eigenvalues have the form $\lambda=\alpha(\tau)+\mathrm{i} \beta(\tau)$, and show that $\alpha(\tau)$ and $\beta(\tau)$ solve the ODEs

$$
\begin{aligned}
& \alpha^{\prime}=\frac{1}{(1+\alpha \tau)^{2}+(\beta \tau)^{2}}\left(\beta^{2}-\alpha^{2}-\alpha \beta^{2} \tau-\alpha^{3} \tau\right), \\
& \beta^{\prime}=-\frac{\beta}{(1+\alpha \tau)^{2}+(\beta \tau)^{2}}\left(2 \alpha+\alpha^{2} \tau+\beta^{2} \tau\right) .
\end{aligned}
$$

We use the discrete set of points defined in (2.9) as initial values in (3.1). Note that if $(\alpha(\tau), \beta(\tau))$ solves (3.1), then $(\alpha(\tau),-\beta(\tau))$ also solves (3.1). Thus, for positive $\tau$ values we restrict our attention to initial values

$$
\alpha\left(\frac{(4 j+1) \pi}{2 K_{2}}\right)=0, \beta\left(\frac{(4 j+1) \pi}{2 K_{2}}\right)=K_{2}, j \geq 0 .
$$

The second step in the proof of Theorem 3.1 is to analyze (3.1)-(3.2) and prove the existence and global behavior of a continuous branch of solutions for each $j \geq 0$. (see Figures 4 and 5). Our method of proof can easily be adapted to prove the existence and qualitative properties of continuous branches of solutions of (3.1) corresponding to the second set of points in (2.9) where $(\alpha, \beta, \tau)=\left(0, \pm K_{2},-\frac{(2 j+2) \pi}{2 K_{2}}\right)$. In Sections 5 and 6 we use these branches to construct families of solutions of the full tumor volume model (1.4) which resolve the issues described in Goal I and Goal II.

Theorem 3.1 (Global Existence and Behavior of Solutions of (3.1)). For each integer $j \geq 0$, there are $\mathrm{C}^{1}$ functions $\alpha_{j}(\tau)$ and $\beta_{j}(\tau)$ which exist for all $\tau$ in a maximal open interval containing $\tau_{j}=\frac{(4 j+1) \pi}{2 K_{2}}$, and satisfy

$$
\begin{aligned}
& \alpha_{j}(\tau)+K_{2} e^{-\alpha_{j}(\tau) \tau} \cos \left(\beta_{j}(\tau) \tau\right)=0, \\
& \beta_{j}(\tau)-K_{2} e^{-\alpha_{j}(\tau) \tau} \sin \left(\beta_{j}(\tau) \tau\right)=0 .
\end{aligned}
$$

At $\tau=\frac{(4 j+1) \pi}{2 K_{2}}$ the solution $\left(\alpha_{j}(\tau), \beta_{j}(\tau)\right)$ satisfies

$$
\left(\alpha_{j}\left(\frac{(4 j+1) \pi}{2 K_{2}}\right), \beta_{j}\left(\frac{(4 j+1) \pi}{2 K_{2}}\right)\right)=\left(0, K_{2}\right) .
$$

Global qualitative properties of solutions of (3.3)-(3.4) are summarized in two cases:

CASE I. Let $j=0$ and define $\tau^{*}=\frac{1}{e K_{2}}$. Then $\alpha_{0}(\tau)$ and $\beta_{0}(\tau)$ exist for all $\tau \in\left(\tau^{*}, \infty\right)$,

$$
\begin{gathered}
\alpha_{0}(\tau)<0 \text { and } \beta_{0}(\tau)>0 \forall \tau \in\left(\tau^{*}, \frac{\pi}{2 K_{2}}\right), \\
0<\alpha_{0}(\tau)<\frac{1}{\tau}\left(\ln (\tau)-\ln \left(\frac{\pi}{2 K_{2}}\right)\right), \frac{\pi}{2 \tau}<\beta_{0}(\tau)<\frac{3 \pi}{2 \tau} \\
\text { and } \beta_{0}^{\prime}(\tau)<0 \forall \tau>\frac{\pi}{2 K_{2}},
\end{gathered}
$$

and

$$
\lim _{\tau \rightarrow \tau^{*+}}\left(\alpha_{0}(\tau), \beta_{0}(\tau)\right)=\left(-e K_{2}, 0\right) \text { and } \lim _{\tau \rightarrow \infty}\left(\alpha_{0}(\tau), \beta_{0}(\tau)\right)=(0,0) .
$$

CASE II. Let $j \geq 1$. Then $\alpha_{j}(\tau)$ and $\beta_{j}(\tau)$ exist for all $\tau \in(0, \infty)$,

$$
\beta_{j}^{\prime}(\tau)<0 \text { and } \frac{2 j \pi}{\tau}<\beta_{j}(\tau)<\frac{(4 j+1) \pi}{2 \tau} \forall \tau \in\left(0, \frac{(4 j+1) \pi}{2 K_{2}}\right),
$$



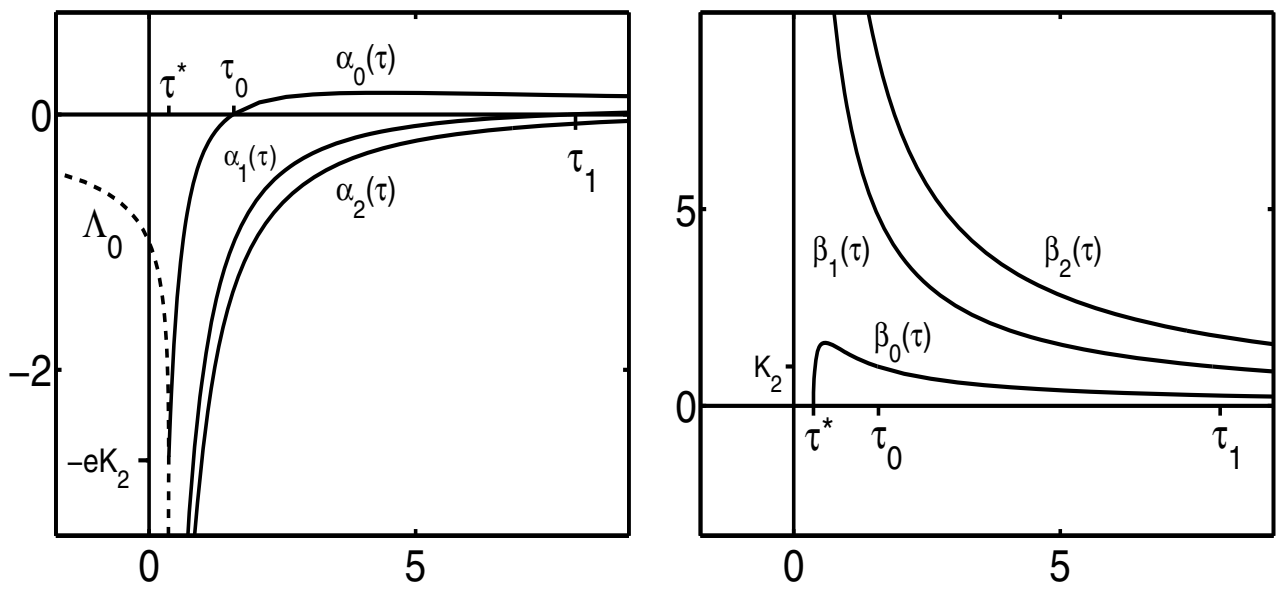

FIG. 4. Left: Graphs of $\alpha_{0}(\tau), \alpha_{1}(\tau), \alpha_{2}(\tau)$ and the real eigenvalue branch $\Lambda_{0}$. Right: Graphs of $\beta_{0}(\tau), \beta_{1}(\tau)$ and $\beta_{2}(\tau)$. Horizontal axes give $\tau$ values; all curves are computed at $K_{2}=1$. The functions $\alpha_{0}(\tau)$ and $\beta_{0}(\tau)$ are bounded. Their interval of existence is $\left[\tau^{*}, \infty\right)=$ $\left[\frac{1}{e}, \infty\right)$. However, $\alpha_{1}(\tau), \alpha_{2}(\tau), \beta_{1}(\tau)$ and $\beta_{2}(\tau)$ are all unbounded and exist on the entire interval $\left(0, \infty\right.$.) Note that $\alpha_{0}\left(\tau_{0}\right)=0$ and $\alpha_{1}\left(\tau_{1}\right)=0$, i.e. the eigenvalues are imaginary at $\tau_{0}=\frac{\pi}{2}$ and $\tau_{1}=$ $\frac{5 \pi}{2}$.

and there is a $\bar{\tau} \in\left(\left(\frac{4 j+1}{2 K_{2}}\right) \pi \mathrm{e}^{-\left(1+\frac{1}{4 j^{2} \pi^{2}}\right)}, \frac{(4 j+1)}{2 K_{2}} \pi\right)$ such that

$$
\alpha_{j}(\tau)<-\frac{1}{\tau} \forall \tau \in(0, \bar{\tau}), \alpha_{j}(\bar{\tau})=-\frac{1}{\bar{\tau}},-\frac{1}{\tau}<\alpha_{j}(\tau)<0 \forall \tau \in\left(\bar{\tau}, \frac{(4 j+1) \pi}{2 K_{2}}\right) .
$$

A uniformly valid lower bound for $\bar{\tau}$ is

$$
\bar{\tau} \geq \frac{5 \pi}{2 K_{2}} e^{-\left(1+\frac{1}{4 \pi^{2}}\right)} \forall j \geq 1 .
$$

When $\tau \geq \frac{(4 j+1) \pi}{2 K_{2}}$ the solution $\left(\alpha_{j}(\tau), \beta_{j}(\tau)\right)$ satisfies

$$
\beta_{j}^{\prime}(\tau)<0 \text { and } \frac{(4 j+1) \pi}{2 \tau} \leq \beta_{j}(\tau)<\frac{(4 j+3) \pi}{2 \tau} \forall \tau \geq \frac{(4 j+1) \pi}{2 K_{2}}
$$

and

$$
0 \leq \alpha_{j}(\tau) \leq \frac{1}{\tau}\left(\ln (\tau)-\ln \left(\frac{(4 j+1) \pi}{2 K_{2}}\right)\right) \forall \tau \geq \frac{(4 j+1) \pi}{2 K_{2}}
$$

REMARKS. Figure 4 illustrates the fundamental differences in the behavior of $\left(\alpha_{j}(\tau)\right.$, $\left.\beta_{j}(\tau)\right)$ between the cases $j=0$ and $j \geq 1$. When $j=0$, the solution $\left(\alpha_{0}(\tau), \beta_{0}(\tau)\right)$ remains bounded over its interval of existence $\left(\tau^{*}, \infty\right)=\left(\frac{1}{e K_{2}}, \infty\right)$. When $j \geq 1$ the interval of existence of $\left(\alpha_{j}(\tau), \beta_{j}(\tau)\right)$ is $(0, \infty)$, but now $\left(\alpha_{j}(\tau), \beta_{j}(\tau)\right)$ is unbounded, with $\alpha_{j}(\tau) \rightarrow-\infty$ and $\beta_{j}(\tau) \rightarrow \infty$ as $\tau \rightarrow 0^{+}$.

Proof of Theorem 3.1. The first part of the proof uses the Implicit Function Theorem applied to (1.7). For this define the functions on the left side of (1.7) by

$$
f(\alpha, \beta, \tau)=\alpha+K_{2} e^{-\alpha \tau} \cos (\beta \tau) \text { and } g(\alpha, \beta, \tau)=\beta-K_{2} e^{-\alpha \tau} \sin (\beta \tau) .
$$



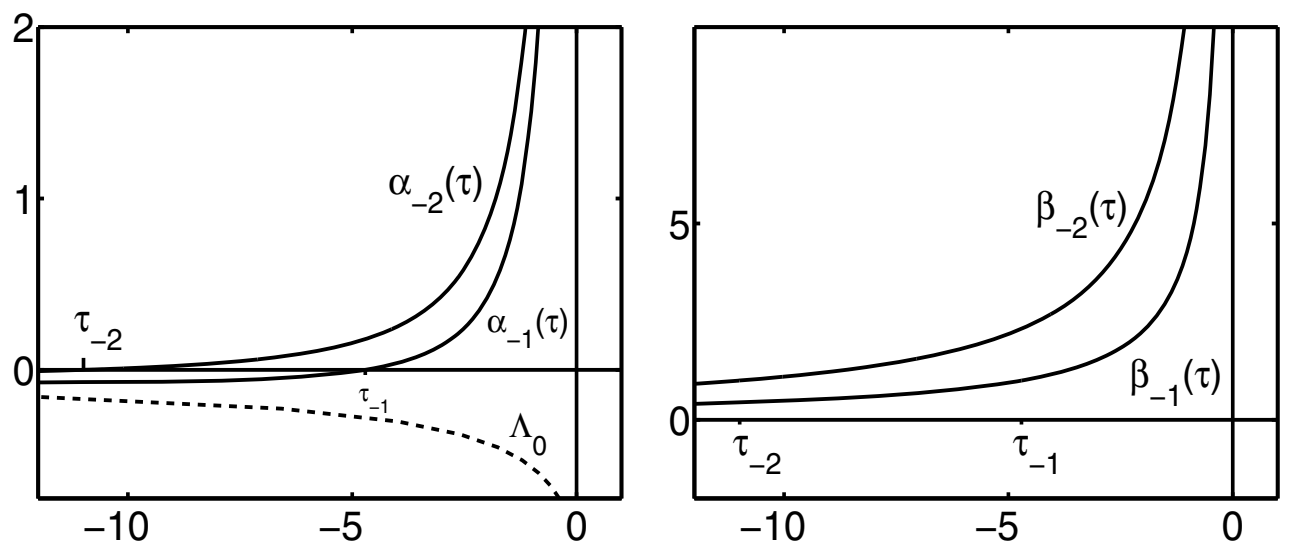

FIG. 5. Left: The graphs of $\alpha_{-1}(\tau), \alpha_{-2}(\tau)$ and the real eigenvalue branch $\Lambda_{0}$. Right: The graphs of $\beta_{-1}(\tau)$ and $\beta_{-2}(\tau)$. All curves computed at $K_{2}=1$. Note that $\left.\alpha_{-2}(\tau), \alpha_{-2}(\tau), \beta_{-1}(\tau)\right)$ and $\beta_{2}(-\tau)$ are all unbounded at $\tau=0$, and exist on the entire interval $(-\infty, 0$.).

For each $j \geq 0$ it is easily verified that $f\left(0, K_{2}, \frac{(4 j+1) \pi}{2 K_{2}}\right)=g\left(0, K_{2}, \frac{(4 j+1) \pi}{2 K_{2}}\right)=0$. The matrix of partial derivatives of $f$ and $g$ with respect to $\alpha$ and $\beta$ satisfies $\operatorname{det}(A) \neq$ 0 when $(\alpha, \beta, \tau)=\left(0, K_{2}, \frac{(4 j+1) \pi}{2 K_{2}}\right), j \geq 0$. Thus, for each $j \geq 0$, the Implicit Function Theorem implies that there is an $\epsilon_{j}>0$ such that if $\tau \in\left(\frac{(4 j+1) \pi}{2 K_{2}}-\epsilon_{j}, \frac{(4 j+1) \pi}{2 K_{2}}+\epsilon_{j}\right)$, then there are unique $C^{1}$ functions $\alpha_{j}(\tau), \beta_{j}(\tau)$ which satisfy system (3.3). For each $j \geq 0$, let $\left(\tau_{\min }(j), \tau_{\max }(j)\right)$ denote the the maximal interval of existence of $\left(\alpha_{j}(\tau), \beta_{j}(\tau)\right)$. To determine $\left(\tau_{\min }(j), \tau_{\max }(j)\right)$ and the behavior of $\alpha_{j}(\tau)$ and $\beta_{j}(\tau)$ on $\left(\tau_{\min }(j), \tau_{\max }(j)\right)$ we derive and analyze related ODEs. It follows from a differentiation of (3.3) that $\alpha_{j}(\tau)$ and $\beta_{j}(\tau)$ satisfy

$$
\begin{aligned}
& \left(1+\alpha_{j}(\tau) \tau\right) \alpha_{j}^{\prime}-\beta_{j} \tau \beta_{j}^{\prime}=\beta_{j}^{2}-\alpha_{j}^{2}, \alpha_{j}\left(\frac{(4 j+1) \pi}{2 K_{2}}\right)=0 \\
& \beta_{j} \tau \alpha_{j}^{\prime}+\left(1+\alpha_{j} \tau\right) \beta_{j}^{\prime}=-2 \alpha_{j} \beta_{j}, \beta_{j}\left(\frac{(4 j+1) \pi}{2 K_{2}}\right)=K_{2} .
\end{aligned}
$$

An algebraic manipulation transforms (3.14) into the nonautonomous, nonlinear ODE system

$$
\begin{aligned}
& \alpha_{j}^{\prime}=\frac{1}{\left(1+\alpha_{j} \tau\right)^{2}+\left(\beta_{j} \tau\right)^{2}}\left(\beta_{j}^{2}-\alpha_{j}^{2}-\alpha_{j} \beta_{j}^{2} \tau-\alpha_{j}^{3} \tau\right), \alpha_{j}\left(\frac{(4 j+1) \pi}{2 K_{2}}\right)=0, \\
& \beta_{j}^{\prime}=-\frac{\beta_{j}}{\left(1+\alpha_{j} \tau\right)^{2}+\left(\beta_{j} \tau\right)^{2}}\left(2 \alpha_{j}+\alpha_{j}^{2} \tau+\beta_{j}^{2} \tau\right), \beta_{j}\left(\frac{(4 j+1) \pi}{2 K_{2}}\right)=K_{2} .
\end{aligned}
$$

Our analysis of (3.15) makes use of the functionals $Q(\tau)=\beta_{j}(\tau) \tau$ and $H(\tau)=\alpha_{j}(\tau) \tau+1$. It follows from (3.15) that $Q$ and $H$ satisfy

$$
\begin{aligned}
& Q^{\prime}=\frac{Q}{\tau\left(H^{2}+Q^{2}\right)}, Q\left(\frac{(4 j+1) \pi}{2 K_{2}}\right)=\frac{(4 j+1) \pi}{2}, \\
& H^{\prime}=\frac{1}{\tau}-\frac{H}{\tau\left(H^{2}+Q^{2}\right)}, H\left(\frac{(4 j+1) \pi}{2 K_{2}}\right)=1 .
\end{aligned}
$$


Below, we show that the qualitative behavior of $\left(\alpha_{j}(\tau), \beta_{j}(\tau)\right)$ is the same for each $j \geq 0$ when $\tau \geq \frac{(4 j+1) \pi}{2 K_{2}}$. However, when $\tau<\frac{(4 j+1) \pi}{2 K_{2}}$ there is a fundamental difference in behavior between the cases $j=0$ and $j \geq 1$. Thus, we divide the remainder of the proof into three parts:

(i) The analysis of $\left(\alpha_{j}(\tau), \beta_{j}(\tau)\right)$ when $\tau \geq \frac{(4 j+1) \pi}{2 K_{2}}$ and $j \geq 0$.

(ii) The analysis of $\left(\alpha_{j}(\tau), \beta_{j}(\tau)\right)$ when $\tau<\frac{(4 j+1) \pi}{2 K_{2}}$ and $j \geq 1$.

(iii) The analysis of $\left(\alpha_{0}(\tau), \beta_{0}(\tau)\right)$ when $\tau<\frac{\pi}{2 K_{2}}$.

Part (i). The analysis of $\left(\alpha_{j}(\tau), \beta_{j}(\tau)\right)$ when $\tau \geq \frac{(4 j+1) \pi}{2 K_{2}}$ and $j \geq 0$.

Our goal here is to show that if $j \geq 0$, then $\tau_{\max }(j)=\infty$ and $\alpha_{j}(\tau), \beta_{j}(\tau)$ satisfy

$$
\beta_{j}^{\prime}(\tau)<0 \text { and } \frac{(4 j+1) \pi}{2 \tau} \leq \beta_{j}(\tau)<\frac{(4 j+3) \pi}{2 \tau} \forall \tau \in\left[\frac{(4 j+1) \pi}{2 K_{2}}, \infty\right)
$$

and

$$
0 \leq \alpha_{j}(\tau) \leq \frac{1}{\tau}\left(\ln (\tau)-\ln \left(\frac{(4 j+1) \pi}{2 K_{2}}\right)\right) \forall \tau \in\left[\frac{(4 j+1) \pi}{2 K_{2}}, \infty\right) .
$$

We begin by assuming that there is a $j \geq 0$ such that $\tau_{\max }(j)<\infty$ and obtain a contradiction. If $\tau_{\max }(j)<\infty$, then standard theory implies that

$$
\limsup _{\tau \rightarrow \tau_{\max }(j)^{-}}\left(\left|\alpha_{j}^{\prime}(\tau)\right|+\left|\beta_{j}^{\prime}(\tau)\right|\right)=\infty
$$

where $\alpha_{j}^{\prime}(\tau)$ and $\beta_{j}^{\prime}(\tau)$ are computed from the ODE system (3.15). To show that (3.19) cannot occur, we use $Q=\beta_{j}(\tau) \tau$. It follows from (3.16) that

$$
Q^{\prime}(\tau)>0 \text { and } Q(\tau) \geq \frac{(4 j+1) \pi}{2} \forall \tau \in\left[\frac{(4 j+1) \pi}{2 K_{2}}, \tau_{\max }(j)\right) .
$$

Substituting $Q=\beta_{j}(\tau) \tau$ into (3.20) gives the lower bound

$$
\beta_{j}(\tau) \geq \frac{(4 j+1) \pi}{2 \tau} \forall \tau \in\left[\frac{(4 j+1) \pi}{2 K_{2}}, \tau_{\max }(j)\right) .
$$

From (3.21) we also conclude that $\beta_{j}(\tau) \geq \frac{\pi}{2 \tau} \forall \tau \in\left[\frac{(4 j+1) \pi}{2 K_{2}}, \tau_{\max }(j)\right)$, hence

$$
\frac{1}{\left(1+\alpha_{j}(\tau) \tau\right)^{2}+\left(\beta_{j}(\tau) \tau\right)^{2}} \leq \frac{4}{\pi^{2}} \forall \tau \in\left[\frac{(4 j+1) \pi}{2 K_{2}}, \tau_{\max }(j)\right)
$$

Next, we derive the lower bound $\alpha_{j}(\tau)>0 \forall \tau \in\left(\frac{(4 j+1) \pi}{2 K_{2}}, \tau_{\max }(j)\right)$. It follows from (3.15) that $\alpha^{\prime}\left(\frac{(4 j+1) \pi}{2 K_{2}}\right)=\left(\frac{2 K_{2}}{(4 j+1) \pi}\right)^{2}>0$. Thus, $\alpha_{j}(\tau)>0$ for small $\tau-\frac{(4 j+1) \pi}{2 K_{2}}>0$. Suppose that there is a $\tilde{\tau} \in\left(\frac{(4 j+1) \pi}{2 K_{2}}, \tau_{\max }(j)\right)$ such that

$$
\alpha_{j}(\tau)>0 \forall \tau \in\left(\frac{(4 j+1) \pi}{2 K_{2}}, \tilde{\tau}\right) \text { and } \alpha_{j}(\tilde{\tau})=0 .
$$

Then the functional $H=\alpha_{j}(\tau) \tau+1$ satisfies

$$
H\left(\frac{(4 j+1) \pi}{2 K_{2}}\right)=1, H(\tau)>1 \forall \tau \in\left(\frac{(4 j+1) \pi}{2 K_{2}}, \tilde{\tau}\right) \text { and } H(\tilde{\tau})=1,
$$


and therefore

$$
H^{\prime}(\tilde{\tau}) \leq 0
$$

However, substituting $\tau=\tilde{\tau}$ into the second equation of (3.16) gives $H^{\prime}(\tilde{\tau})=\frac{Q^{2}(\tilde{\tau})}{\tilde{\tau}\left(1+Q^{2}(\tilde{\tau})\right)}>$ 0 , contradicting (3.25). We conclude that

$$
\alpha_{j}(\tau)>0 \forall \tau \in\left(\frac{(4 j+1) \pi}{2 K_{2}}, \tau_{\max }(j)\right) .
$$

Next, it follows from (3.3) that

$$
\cos \left(\beta_{j}(\tau) \tau\right)=-\frac{\alpha_{j}(\tau)}{K_{2}} e^{\alpha_{j}(\tau) \tau} \text { and } \sin \left(\beta_{j}(\tau) \tau\right)=\frac{\beta_{j}(\tau)}{K_{2}} e^{\alpha_{j}(\tau) \tau} .
$$

Combining (3.21), (3.26) and (3.27) implies that $\cos \left(\beta_{j}(\tau) \tau\right)<0 \forall \tau \in\left(\frac{(4 j+1) \pi}{2 K_{2}}, \tau_{\max }(j)\right)$, hence

$$
\frac{(4 j+1) \pi}{2 \tau} \leq \beta_{j}(\tau)<\frac{(4 j+3) \pi}{2 \tau} \forall \tau \in\left[\frac{(4 j+1) \pi}{2 K_{2}}, \tau_{\max }(j)\right) .
$$

We also conclude from (3.15), (3.26) and (3.28) that

$$
\beta_{j}^{\prime}(\tau)<0 \forall \tau \in\left[\frac{(4 j+1) \pi}{2 K_{2}}, \tau_{\max }(j)\right) .
$$

REMARK. The derivation of (3.26), (3.28) and (3.29) is the same when $\tau_{\max }=\infty$. This proves (3.17) and the lower bound in (3.18). Next, it follows from (3.27) that

$$
1=\sin ^{2}\left(\beta_{j}(\tau) \tau\right)+\cos ^{2}\left(\beta_{j}(\tau) \tau\right)=\frac{\alpha_{j}^{2}(\tau)+\beta_{j}^{2}(\tau)}{K_{2}^{2}} e^{2 \alpha_{j}(\tau) \tau} .
$$

This, and the fact that $\alpha_{j}(\tau)>0$ when $\tau>\frac{(4 j+1) \pi}{2 K_{2}}$, imply that

$$
\alpha_{j}^{2}(\tau)+\beta_{j}^{2}(\tau) \leq K_{2}^{2} \forall \tau \in\left[\frac{(4 j+1) \pi}{2 K_{2}}, \tau_{\max }(j)\right) .
$$

Thus, from (3.22), (3.31) and the first equation in (3.15) we conclude that

$$
\left|\alpha_{j}^{\prime}(\tau)\right| \leq \frac{4}{\pi^{2}}\left(\alpha_{j}^{2}(\tau)+\beta_{j}^{2}(\tau)\right)\left(1+\left|\alpha_{j}(\tau)\right| \tau\right) \leq \frac{4 K_{2}^{2}}{\pi^{2}}\left(1+K_{2} \tau_{\max }(j)\right) .
$$

Similarly, it follows from (3.31) and the second equation in (3.15) that

$$
\left|\beta_{j}^{\prime}(\tau)\right| \leq \frac{4\left|\beta_{j}(\tau)\right|}{\pi^{2}}\left(\left|\alpha_{j}(\tau)\right|\left(2+\left|\alpha_{j}(\tau)\right| \tau\right)+\beta_{j}^{2}(\tau) \tau\right) \leq \frac{4 K_{2}^{2}}{\pi^{2}}\left(2+K_{2} \tau_{\max }(j)\right) .
$$

Thus, both $\alpha_{j}^{\prime}(\tau)$ and $\beta_{j}^{\prime}(\tau)$ are bounded on $\left[\frac{(4 j+1) \pi}{2 K_{2}}, \tau_{\max }(j)\right)$, hence (3.19) cannot hold for any $j \geq 0$. We conclude that $\tau_{\max }(j)=\infty \forall j \geq 0$ as claimed.

Finally, we derive the upper bound for $\alpha_{j}(\tau)$ given in (3.18). First, it follows from (3.26) that $H=\alpha_{j}(\tau) \tau+1 \geq 1 \forall \tau \geq \frac{(4 j+1) \pi}{2 K_{2}}$. From this and the second equation in (3.16) we conclude that

An integration gives

$$
H^{\prime}(\tau) \leq \frac{1}{\tau} \forall \tau \in\left[\frac{(4 j+1) \pi}{2 K_{2}}, \infty\right) .
$$

$$
1<H(\tau)<1+\ln (\tau)-\ln \left(\frac{(4 j+1) \pi}{2 K_{2}}\right) \forall \tau \in\left(\frac{(4 j+1) \pi}{2 K_{2}}, \infty\right) .
$$


Substituting $H=\alpha_{j}(\tau) \tau+1$ into (3.35), we obtain

$$
0<\alpha_{j}(\tau)<\frac{1}{\tau}\left(\ln (\tau)-\ln \left(\frac{(4 j+1) \pi}{2 K_{2}}\right)\right) \forall \tau \in\left(\frac{(4 j+1) \pi}{2 K_{2}}, \infty\right) .
$$

This completes the proof of (3.17)-(3.18).

Part (ii). The analysis of $\left(\alpha_{j}(\tau), \beta_{j}(\tau)\right)$ when $\tau \leq \frac{(4 j+1) \pi}{2 K_{2}}$, for $j \geq 1$. In this part we let $j \geq 1$ and prove that $\tau_{\min }(j)=0$, that $\alpha_{j}(\tau), \beta_{j}(\tau)$ satisfy

$$
\beta_{j}^{\prime}(\tau)<0 \text { and } \frac{2 j \pi}{\tau}<\beta_{j}(\tau) \leq \frac{(4 j+1) \pi}{2 \tau} \forall \tau \in\left(0, \frac{(4 j+1) \pi}{2 K_{2}}\right],
$$

and that there exists a $\bar{\tau} \in\left(0, \frac{(4 j+1) \pi}{2 K_{2}}\right)$ such that

$$
\alpha_{j}(\tau)<-\frac{1}{\tau} \forall \tau \in(0, \bar{\tau}), \alpha_{j}(\bar{\tau})=-\frac{1}{\bar{\tau}},-\frac{1}{\tau}<\alpha_{j}(\tau)<0 \forall \tau \in\left(\bar{\tau}, \frac{(4 j+1) \pi}{2 K_{2}}\right) .
$$

The first step is to assume that there is a $j \geq 1$ such that $\tau_{\min }(j) \neq 0$, and obtain a contradiction. The first possibility to eliminate is that

$$
\tau_{\min }(j)>0 .
$$

It follows from (3.16) and uniqueness of the solution $Q=0$ that

$$
\begin{array}{r}
Q^{\prime}(\tau)>0 \text { and } 0<Q(\tau)=\beta_{j}(\tau) \tau \leq Q\left(\frac{(4 j+1) \pi}{2 K_{2}}\right)=\frac{(4 j+1) \pi}{2} \\
\forall \tau \in\left(\tau_{\min }(j), \frac{(4 j+1) \pi}{2 K_{2}}\right] .
\end{array}
$$

We claim that sharper bounds on $Q(\tau)$ are given by

$$
2 j \pi<Q(\tau) \leq \frac{(4 j+1) \pi}{2} \forall \tau \in\left(\tau_{\min }(j), \frac{(4 j+1) \pi}{2 K_{2}}\right] .
$$

If the lower bound in (3.41) is false, then there exists $\hat{\tau} \in\left(\tau_{\min }(j), \frac{(4 j+1) \pi}{2 K_{2}}\right)$ such that $Q(\hat{\tau})=2 j \pi$. Setting $\tau=\hat{\tau}$ in the second equation of (3.3) gives $\beta_{j}(\hat{\tau})=0$, hence $Q(\hat{\tau})=\beta_{j}(\hat{\tau}) \hat{\tau}=0$, which contradicts (3.40). Thus, $\hat{\tau}$ does not exist and (3.41) is proved.

Next, since we are assuming that $\tau_{\min }(j)>0$ is finite, then standard theory implies that

$$
\limsup _{\tau \rightarrow \tau_{\min }(j)^{+}}\left(\left|\alpha_{j}^{\prime}(\tau)\right|+\left|\beta_{j}^{\prime}(\tau)\right|\right)=\infty .
$$

Because of (3.42), the term $\left.\left(1+\alpha_{j}(\tau) \tau\right)^{2}+\left(\beta_{j}(\tau) \tau\right)^{2}\right)$ in (3.15) satisfies

$$
\left.\liminf _{\tau \rightarrow \tau_{\min }(j)^{+}}\left(\left(\alpha_{j}(\tau) \tau+1\right)^{2}+\left(\beta_{j}(\tau) \tau\right)^{2}\right)\right)=0 .
$$

This implies that there is a positive, decreasing sequence $\left\{\tau^{j}\right\}$ such that

$$
\tau^{j} \rightarrow \tau_{\min }(j)^{+} \text {and } Q\left(\tau^{j}\right)=\beta_{j}\left(\tau^{j}\right) \tau^{j} \rightarrow 0 \text { as } j \rightarrow \infty .
$$

Thus, $Q\left(\tau^{j}\right)<2 j \pi$ for large $j$, which contradicts (3.41). This eliminates possibility (3.39) and we conclude that $\tau_{\min }(j) \leq 0 \forall j \geq 1$. Suppose that $\tau_{\min }(j)<0$ for some $j \geq 1$. Setting $\tau=0$ in the second equation of (3.3) gives $\beta_{j}(0)=0$, which contradicts (3.40). Thus, we conclude that $\tau_{\min }(j)=0 \forall j \geq 1$, as claimed. 
Next, we prove that $\beta_{j}^{\prime}<0$, and the bounds on $\alpha_{j}(\tau)$ in (3.38). First, (3.3) and (3.37) imply that

$$
\alpha_{j}(\tau)=-K_{2} e^{-\alpha_{j}(\tau) \tau} \cos \left(\beta_{j}(\tau) \tau\right)<0 \forall \tau \in\left(0, \frac{(4 j+1) \pi}{2 K_{2}}\right) .
$$

Second, we need to show that

$$
\alpha_{j}^{2}(\tau) \tau+2 \alpha_{j}(\tau)+\beta_{j}^{2}(\tau) \tau>0 \forall \tau \in\left(0, \frac{(4 j+1) \pi}{2 K_{2}}\right] .
$$

When $\tau=\frac{(4 j+1) \pi}{2 K_{2}}$ the left side of (3.46) reduces to $\frac{(4 j+1) K_{2} \pi}{2}>0$. Also, (3.46) is a quadratic in $\tau$. Thus, since $\beta_{j} \tau>2 j \pi$, the discriminant $D$ of (3.46) satisfies

$$
D=4-4 \beta_{j}^{2} \tau^{2} \leq 4-4(2 j \pi)^{2}<0 \forall j \geq 1 .
$$

This implies that (3.46) holds. Finally, from (3.15), (3.45), (3.46) and the lower bound $\beta_{j}(\tau)>0$, we conclude that $\beta_{j}^{\prime}<0 \forall \tau \in\left(0, \frac{(4 j+1) \pi}{2 K_{2}}\right)$. This completes the proof of (3.37).

It remains to prove (3.38). The first step is to show that there is a $\bar{\tau} \in\left(0, \frac{(4 j+1) \pi}{2 K_{2}}\right)$ such that $\alpha_{j}(\bar{\tau})=-\frac{1}{\tau}$. Suppose that there is a $j \geq 1$ for which such a $\bar{\tau}$ does not exist. Then

$$
H(\tau)=\alpha_{j}(\tau) \tau+1 \in(0,1) \forall \tau \in\left(0, \frac{(4 j+1) \pi}{2 K_{2}}\right) \text { and } H\left(\frac{(4 j+1) \pi}{2 K_{2}}\right)=1 .
$$

This, (3.45) and the lower bound $Q(\tau)>2 j \pi$ imply that the term $\frac{H}{\tau\left(H^{2}+Q^{2}\right)}$, which is an increasing function of $H$, satisfies

$$
0<\frac{H}{\tau\left(H^{2}+Q^{2}\right)}<\frac{1}{\tau\left(1+4 j^{2} \pi^{2}\right)} \forall \tau \in\left(0, \frac{(4 j+1) \pi}{2 K_{2}}\right) .
$$

Substituting (3.49) into the second equation in (3.16) gives

$$
H^{\prime} \geq \frac{1}{\tau}-\frac{1}{\tau\left(1+4 j^{2} \pi^{2}\right)}=\frac{4 j^{2} \pi^{2}}{\tau\left(1+4 j^{2} \pi^{2}\right)} \forall \tau \in\left(0, \frac{(4 j+1) \pi}{2 K_{2}}\right) .
$$

Integrating (3.50) from $\tau$ to $\frac{(4 j+1) \pi}{2 K_{2}}$ gives

$$
H(\tau) \leq 1+\frac{4 j^{2} \pi^{2}}{\left(1+4 j^{2} \pi^{2}\right)}\left(\ln (\tau)-\ln \left(\frac{(4 j+1) \pi}{2 K_{2}}\right)\right) \forall \tau \in\left(0, \frac{(4 j+1) \pi}{2 K_{2}}\right] .
$$

The right side of (3.51) is negative when $\tau=\left(\frac{4 j+1}{2 K_{2}}\right) \pi \mathrm{e}^{-\left(1+\frac{1}{4 j^{2} \pi^{2}}\right)}$. Thus, we conclude that there is a

$$
\bar{\tau} \in\left(\left(\frac{4 j+1}{2 K_{2}}\right) \pi \mathrm{e}^{-\left(1+\frac{1}{4 j^{2} \pi^{2}}\right)}, \frac{(4 j+1)}{2 K_{2}} \pi\right)
$$

such that

$$
H(\bar{\tau})=0 \text { and } H(\tau)>0 \forall \tau \in\left(\bar{\tau}, \frac{(4 j+1)}{2 K_{2}} \pi\right] .
$$

It follows from (3.52) that a uniformly valid lower bound for $\bar{\tau}$ is

$$
\bar{\tau} \geq \frac{5 \pi}{2 K_{2}} e^{-\left(1+\frac{1}{4 \pi^{2}}\right)} \forall j \geq 1 \text {. }
$$


This proves the lower bound (3.10) in the statement of the theorem. Finally, an integration of the second equation in (3.16) from $\tau$ to $\bar{\tau}$ gives

$$
H(\tau)=-\int_{\tau}^{\bar{\tau}} \frac{1}{x} \exp \left(\int_{\tau}^{x} \frac{d \eta}{\eta\left(H^{2}(\eta)+Q^{2}(\eta)\right)}\right) d x<0 \forall \tau \in(0, \bar{\tau}) .
$$

Since $H=\alpha_{j}(\tau) \tau+1$, it follows from (3.53)-(3.55) that $\alpha_{j}(\tau)$ satsifes (3.38). This completes the proof of (3.37)-(3.38).

Part (iii). The analysis of $\left(\alpha_{0}(\tau), \beta_{0}(\tau)\right)$ when $\tau \leq \frac{\pi}{2 K_{2}}$. In this part we let $j=0$ and prove that $\tau_{\min }(0)=\tau^{*}=\frac{1}{e K_{2}}$, and that $\alpha_{0}(\tau), \beta_{0}(\tau)$ satisfy

$$
\begin{gathered}
\alpha_{0}(\tau)<0 \text { and } \beta_{0}(\tau)>0 \forall \tau \in\left(\frac{1}{e K_{2}}, \frac{\pi}{2 K_{2}}\right), \\
\lim _{\tau \rightarrow \tau^{*+}}\left(\alpha_{0}(\tau), \beta_{0}(\tau)\right)=\left(-e K_{2}, 0\right) .
\end{gathered}
$$

We begin by assuming that $\tau_{\min }(0) \neq \frac{1}{e K_{2}}$ and obtain a contradiction. The first possibility is

$$
\tau_{\min }(0) \leq 0 .
$$

We make use of the functional $Q=\beta_{0}(\tau) \tau$, which satisfies

$$
Q^{\prime}=\frac{Q}{\tau\left(H^{2}+Q^{2}\right)}, Q\left(\frac{\pi}{2 K_{2}}\right)=\frac{\pi}{2} .
$$

From (3.58) and (3.59) we conclude that there is a $\bar{Q} \in\left[0, \frac{\pi}{2}\right)$ such that

$$
Q^{\prime}(\tau)>0 \text { and } 0<Q(\tau)<\frac{\pi}{2} \forall \tau \in\left(0, \frac{\pi}{2 K_{2}}\right) \text {, and } \lim _{\tau \rightarrow 0^{+}} Q(\tau)=\bar{Q} .
$$

Next, it follows from (3.3) that $\alpha_{0}(\tau)$ and $\beta_{0}(\tau)$ satisfy

$$
\begin{aligned}
& \alpha_{0}(\tau)+K_{2} e^{-\alpha_{0}(\tau) \tau} \cos \left(\beta_{0}(\tau) \tau\right)=0, \\
& \beta_{0}(\tau)-K_{2} e^{-\alpha_{0}(\tau) \tau} \sin \left(\beta_{0}(\tau) \tau\right)=0 .
\end{aligned}
$$

From (3.60) and the second equation in (3.61) we conclude that

$$
\lim _{\tau \rightarrow 0^{+}} \tau K_{2} e^{-\alpha_{0}(\tau) \tau}=\lim _{\tau \rightarrow 0^{+}} \frac{Q(\tau)}{\sin (Q(\tau))}=\frac{\bar{Q}}{\sin (\bar{Q})}=\delta>0 .
$$

Thus,

which in turn implies that

$$
e^{-\alpha_{0}(\tau) \tau} \sim \frac{\delta}{\tau K_{2}} \text { as } \tau \rightarrow 0^{+}
$$

$$
\lim _{\tau \rightarrow 0^{+}} \alpha_{0}(\tau) \tau=-\infty
$$

Next, combine the two equations in (3.61) and conclude from (3.60) and (3.62) that

$$
\lim _{\tau \rightarrow 0^{+}} \frac{-1}{\alpha_{0}(\tau) \tau}=\lim _{\tau \rightarrow 0^{+}} \frac{\tan (Q(\tau))}{Q(\tau)}=\frac{\tan (\bar{Q})}{\bar{Q}}=\gamma>0 .
$$

Therefore

$$
\lim _{\tau \rightarrow 0^{+}}\left|\alpha_{0}(\tau) \tau\right|=\frac{1}{\gamma}<\infty
$$

which contradicts (3.64). Thus, our assumption in (3.58) that $\tau_{\min }(0) \leq 0$ has led to a contradiction. We conclude that $\tau_{\min }(0)>0$ as claimed. 
It remains to prove that $\tau_{\min }(0)=\frac{1}{e K_{2}}$. Since $\tau_{\min }(0)>0$ is finite, then standard theory implies that

$$
\limsup _{\tau \rightarrow \tau_{\min }(0)^{+}}\left(\left|\alpha_{0}^{\prime}(\tau)\right|+\left|\beta_{0}^{\prime}(\tau)\right|\right)=\infty
$$

where $\alpha_{0}^{\prime}(\tau)$ and $\beta_{0}^{\prime}(\tau)$ are computed from the ODE system

$$
\begin{aligned}
& \alpha_{0}^{\prime}=\frac{1}{\left(1+\alpha_{0} \tau\right)^{2}+\left(\beta_{0} \tau\right)^{2}}\left(\beta_{0}^{2}-\alpha_{0}^{2}-\alpha_{0} \beta_{0}^{2} \tau-\alpha_{0}^{3} \tau\right), \alpha_{0}\left(\frac{\pi}{2 K_{2}}\right)=0, \\
& \beta_{0}^{\prime}=-\frac{\beta_{0}}{\left(1+\alpha_{0} \tau\right)^{2}+\left(\beta_{0} \tau\right)^{2}}\left(2 \alpha_{0}+\alpha_{0}^{2} \tau+\beta_{0}^{2} \tau\right), \beta_{0}\left(\frac{\pi}{2 K_{2}}\right)=K_{2} .
\end{aligned}
$$

Because of (3.67), the term $\left(1+\alpha_{0}(\tau) \tau\right)^{2}+\left(\beta_{0}(\tau) \tau\right)^{2}$ in (3.68) must come arbitrarily close to zero as $\tau \rightarrow \tau_{\min }(0)^{+}$. That is,

$$
\liminf _{\tau \rightarrow \tau_{\min }(0)^{+}}\left(\left(\alpha_{0}(\tau) \tau+1\right)^{2}+\left(\beta_{0}(\tau) \tau\right)^{2}\right)=0 .
$$

Since $Q(\tau)=\beta_{0}(\tau) \tau$ is a positive increasing function on $\left(\tau_{\min }(0), \frac{\pi}{2 K_{2}}\right)$, it follows from (3.69) that

$$
\lim _{\tau \rightarrow \tau_{\min }(0)^{+}} Q(\tau)=\lim _{\tau \rightarrow \tau_{\min }(0)^{+}} \beta_{0}(\tau) \tau=0 .
$$

This, and the fact that $\tau_{\min }(0)>0$, imply that

$$
\lim _{\tau \rightarrow \tau_{\min }(0)^{+}} \beta_{0}(\tau)=0 .
$$

Next, combine the two equations in (3.61) and conclude from (3.70) that

$$
-\frac{1}{\alpha_{0}(\tau) \tau}=\frac{\tan (Q(\tau))}{Q(\tau)} \rightarrow 1 \text { as } \tau \rightarrow \tau_{\min }(0)^{+}
$$

From (3.72) and the first equation in (3.61) it follows that

$$
\alpha_{0}(\tau)=-K_{2} e^{-\alpha_{0}(\tau) \tau} \cos (Q(\tau))<0 \forall \tau \in\left(\tau_{\min }(0), \frac{\pi}{2 K_{2}}\right)
$$

and $\lim _{\tau \rightarrow \tau_{\min }(0)} \alpha_{0}(\tau)=-K_{2} e$. Combining these properties with (3.72) gives $\tau_{\min }(0)=$ $\frac{1}{e K_{2}}$. This, (3.71) and (3.73) imply that

$$
\lim _{\tau \rightarrow \tau^{*}}\left(\alpha_{0}(\tau), \beta_{0}(\tau)\right)=\left(-K_{2} e, 0\right) .
$$

This completes the proof of (3.56)-(3.57).

4. The effects of host-tumor interactions. In this section we address the issues in Goal I (see Section 1). We show how our theoretical results can lead to new understandings of host-tumor interactions effects in the absence of external therapy. Of fundamental importance is the parameter, $\tau \geq 0$, which we interpret to represent a delay in the time of response of the inhibitory variable, $G(t)$. We address the following:

(I) Develop methods to analytically show whether, for large periods of time, tumor volume $N(t)$ persists at a detectable level, regresses to an undetectable level, or whether $N(t)$ can fluctuate indefinitely between these states.

(II) For small $\tau \in\left(0, \tau^{*}\right)=\left(0, \frac{1}{e K_{2}}\right)$, why do host-tumor interactions result in tumor growth?

(III) When $\tau^{*}<\tau \leq \tau_{\text {crit }}=\frac{\pi}{2 K_{2}}$, how can host-tumor interactions affect tumor persistence or regression? 
(IV) When $\tau^{*}<\tau \leq \tau_{\text {crit }}$, how can host-tumor interactions result in a dormant state?

(V) When $\tau>\tau_{\text {crit }}$, how can tumor volume exhibit runaway growth?

(I) Analytic methods. To resolve (I)-(V) within the framework of our generalized Gompertzian model we develop methods to characterize both transient and limiting behaviors of solutions. We let $\theta>0$ denote the level of detection of a tumor. Its value decreases as detection techniques improve. Because detection techniques have improved drastically, some tumors which previously had been undetectable, and which subsequently would have regressed, are now treated using more aggressive local therapies such as mastectomy. Such treatment strategies have not been shown to be associated with improved survival 12, 14,26, 44,45]. Furthermore, it has recently been asserted that there is high probability that some cancer patients with small tumors are overtreated [8]. However, small tumors are associated with cell types which can occasionally develop into metastatic cancer. In this situation, systemic treatment (chemotherapy, hormonal therapy) would be appropriate to treat such cancers. Thus, it is important to characterize tumor volume behavior in terms of the inhibitory response $G(t)$.

General form of $G(t)$. Recall from Theorem 3.1 that there are infinitely many eigenvalue solutions $\lambda_{i}=\alpha_{i}(\tau)+i \beta_{i}(\tau)$ of the transcendental equation $\lambda=-K_{2} e^{-\lambda \tau}$ derived in Section 1 (see eq. (1.6) ). Thus, $G(t)$ can be expressed in terms of corresponding eigenfunctions, and system (1.4) becomes

$$
\begin{aligned}
& \frac{d N(t)}{d t}=K_{1} N(t) G(t), N(0)=N_{0}>0, \\
& G(t)=C_{1} e^{r_{1}(\tau) t}+C_{2} e^{r_{2}(\tau) t} \\
& +\sum_{j \in I_{1}} c_{j} \exp \left(\alpha_{j}(\tau) t\right) \cos \left(\beta_{j}(\tau) t\right)+\sum_{k \in I_{2}} d_{k} \exp \left(\alpha_{k}(\tau) t\right) \sin \left(\beta_{k}(\tau) t\right) .
\end{aligned}
$$

$G(t)$ describes the internal response of the host to the presence of the tumor. This response is determined by the collective activity of a large number of individual cells. The second equation of (4.1) posits that the response of each cell group is characterized by eigenfunctions $C_{1} e^{r_{1}(\tau) t}, C_{2} e^{r_{2}(\tau) t}, c_{j} \exp \left(\alpha_{j}(\tau) t\right) \cos \left(\beta_{j}(\tau) t\right)$ or $d_{k} \exp \left(\alpha_{k}(\tau) t\right)$ $\sin \left(\beta_{k}(\tau) t\right)$. The coefficients denote strength of response of the cell group to the tumor. The term $\exp \left(\alpha_{j}(\tau) t\right)$ gives the natural growth rate, or decay rate, of the cell's activity. The function, $\cos \left(\beta_{j}(\tau) t\right)$, represents the characteristic oscillatory component of the cell's activity, with natural frequency $\frac{2 \pi}{\beta_{j}}$. A similar interpretation applies to $d_{k} \exp \left(\alpha_{k}(\tau) t\right) \sin \left(\beta_{k}(\tau) t\right)$. The product $N(t) G(t)$ in (4.1) describes the interaction between the internal response of the host with the tumor.

(II) Classical Gompertzian growth when $0<\tau \ll \tau^{*}=\frac{1}{e K_{2}}$. We prove that tumor growth exhibits the same classical Gompertzian growth that occurs at $\tau=0$ (see Sec. 1 ). The first step is to determine the relative sizes of the real parts of the eigenvalues in the $G(t)$ component of (4.1) when $0<\tau \ll \tau^{*}$. It follows from Case I of Theorem 3.1 that $\alpha_{0}(\tau)$ does not exist when $\tau<\tau^{*}$. From (3.9)-3.10) in Theorem 3.1 we conclude that $\alpha_{j}(\tau) \leq-\frac{1}{\tau} \forall \tau \in\left(0, \frac{1}{e K_{2}}\right), j \geq 1$. Thus,

$$
\alpha_{j}(\tau) \rightarrow-\infty \text { as } \tau \rightarrow 0^{+} \text {uniformly with respect to } j \geq 1
$$


It is easily shown that the real eigenvalues $r_{1}(\tau)$ and $r_{2}(\tau)$ satisfy

$$
\begin{gathered}
r_{2}(\tau) \ll r_{1}(\tau)<0 \text { and } e^{r_{2}(\tau) t} \ll e^{r_{1}(\tau) t} \text { when } 0<\tau \ll \tau^{*}, \\
r_{2}(\tau) \rightarrow-\infty \text { and } r_{1}(\tau) \rightarrow-K_{2} \text { as } \tau \rightarrow 0^{+} .
\end{gathered}
$$

It follows from (4.2)-(4.4) that the eigenfunctions in the $G(t)$ component of (4.1) satisfy

$$
\left|\exp \left(\alpha_{j}(\tau) t\right) \cos \left(\beta_{j}(\tau) t\right)\right| \ll e^{r_{1}(\tau) t} \text { and }\left|\exp \left(\alpha_{k}(\tau) t\right) \sin \left(\beta_{k}(\tau) t\right)\right| \ll e^{r_{1}(\tau) t},
$$

for small $\tau>0$, uniformly with respect to $j \geq 1$ and $k \geq 1$. These effects become more pronounced as $t$ increases. For small $\tau>0$, these properties suggest that the second term in the $G(t)$ component of (4.1), as well as the terms in the summation, have an insignificant effect on the behavior of $G(t)$ in comparison to the first term. Thus, when $\tau>0$ is small, the $G(t)$ component of (4.1) reduces to $G=G_{1} e^{r_{1}(\tau) t}$. Substituting this function gives

$$
\frac{d N(t)}{d t}=K_{1} N(t) G_{1} e^{r_{1}(\tau) t}, N(0)=N_{0}>0,
$$

where $0<\tau \ll \tau^{*}$ and $r_{1} \approx-K_{2}$. The solution of (4.6) is

$$
N(t)=N_{0} \exp \left(\frac{K_{1} c_{1}}{r_{1}}\left(e^{r_{1} t}-1\right)\right), t \geq 0 .
$$

We conclude from (4.7) that, when $G_{1}>0, N(t)$ increases as $t$ increases and exhibits the same classical Gompertzian growth pattern as in the original model (1.2) where $\tau=0$.

(III) Tumor regression when $\tau^{*}<\tau \leq \tau_{\text {crit }}=\frac{\pi}{2 K_{2}}$. Figure 6 demonstrates tumor regression for the parameter set $\left(K_{1}, K_{2}, N_{0}, \theta\right)=(1,1, .01,13)$ and $\tau=\tau_{\text {crit }}=\frac{\pi}{2}$. When $\tau=\frac{\pi}{2}$, Theorem 3.1 shows that there are no real eigenvalues, and infinitely many complex eigenvalues, one of which is purely imaginary. We solve (4.1) using a three-term expansion for $G(t)$ based on the eigenvalues

$$
\alpha_{0}\left(\frac{\pi}{2}\right)+\mathrm{i} \beta_{0}\left(\frac{\pi}{2}\right), \alpha_{1}\left(\frac{\pi}{2}\right)+\mathrm{i} \beta_{1}\left(\frac{\pi}{2}\right) \text { and } \alpha_{2}\left(\frac{\pi}{2}\right)+\mathrm{i} \beta_{2}\left(\frac{\pi}{2}\right) .
$$

Graphs of $\alpha_{0}(\tau), \alpha_{1}(\tau), \alpha_{2}(\tau), \beta_{0}(\tau), \beta_{1}(\tau)$ and $\beta_{1}(\tau)$ are shown in Figure 4 Thus,

$$
G(t)=c_{0} \cos (t)+c_{1} e^{\alpha_{1}\left(\frac{\pi}{2}\right) t} \cos \left(\beta_{1}\left(\frac{\pi}{2}\right) t\right)+c_{2} e^{\alpha_{2}\left(\frac{\pi}{2}\right) t} \cos \left(\beta_{2}\left(\frac{\pi}{2}\right) t\right),
$$

which is a solution of $G^{\prime}(t)=G\left(t-\frac{\pi}{2}\right)$. Substituting (4.9) into the first equation of (1.4) gives

$$
N^{\prime}(t)=N(t)\left(c_{0} \cos (t)+c_{1} e^{\alpha_{1}\left(\frac{\pi}{2}\right) t} \cos \left(\beta_{1}\left(\frac{\pi}{2}\right) t\right)+c_{2} e^{\alpha_{2}\left(\frac{\pi}{2}\right) t} \cos \left(\beta_{2}\left(\frac{\pi}{2}\right) t\right)\right) .
$$

Figure 6] shows the solution of (4.9)-(4.10) when $c_{0}=-20, c_{1}=-11$ and $c_{2}=9$. The left panel shows that tumor volume becomes detectable at $t \approx .9$, then oscillates around the level of detection $\theta$, and ultimately regresses into a state of undectability where $N(t)<\theta$. The results shown in Figure 6 demonstrate that host-tumor interactions by themselves can cause the tumor to regress.

(IV) Tumor dormancy. Figure 7 illustrates how tumor dormancy can result from hosttumor interactions. Here we use the same three-term expansion for $G(t)$ as in Figure 6 , but with the coefficients changed to $c_{0}=8.5$ and $c_{1}=c_{2}=0$. In this case tumor volume becomes detectable at $t \approx 1$, but then regresses to a dormant state, where $N(t)<\theta$, at 

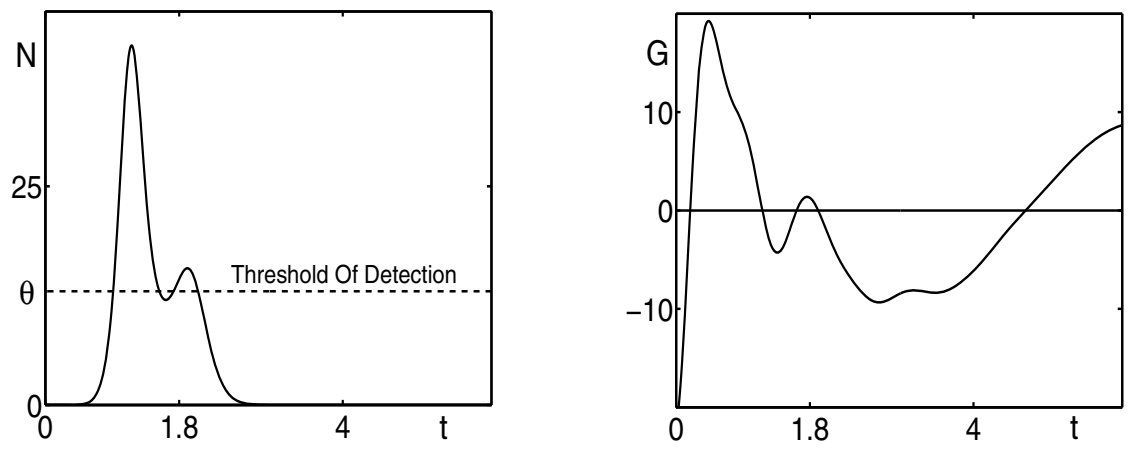

FIG. 6. Tumor Regression. $N(t)$ and $G(t)$ are computed from (4.9) and (4.10) where $G(t)$ has a three-term expansion, and the eigenvalues are complex. The parameters are $\left(K_{1}, N(0), K_{2}, \tau, \theta\right)=$ $\left(1,1, .01, \frac{\pi}{2}, 13\right)$. For the choice $\left(c_{0}, c_{1}, c_{2}\right)=(-20,-11,9)$ tumor volume becomes detectable when $t \approx .9$, then oscillates around the level of detection $\theta$, and ultimately regresses into a state of undetectability where $N(t)<\theta$.
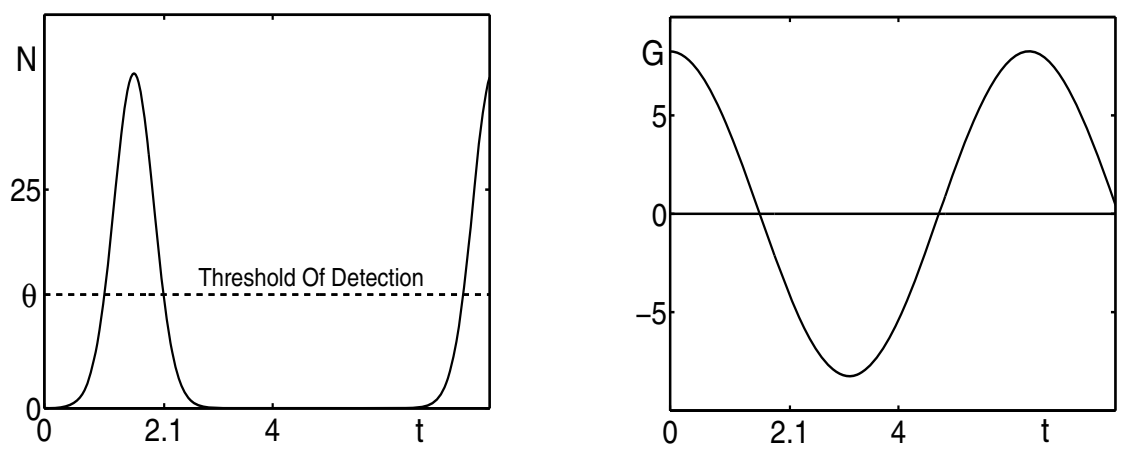

FIG. 7. Tumor dormancy. $N(t)$ and $G(t)$ are computed using the same same parameter values, and three-term expansion for $G(t)$, as in Figure 6] For the choice $\left(c_{0}, c_{1}, c_{2}\right)=(-20,-11,7)$ tumor volume becomes detectable at $t \approx 1$, then regresses into a state of dormancy, where $N(t)<\theta$, at $t \approx 2.1$. Subsequently, $N(t)<\theta$ and tumor volume remains in the dormant state until $t \approx 8$, where $N(t)$ again exceeds the detectable level and regrowth occurs.

$t \approx 2.1$. Subsequently, $N(t)<\theta$ and tumor volume remains in the dormant state until $t \approx 8$, where $N(t)$ again exceeds the detectable level and regrowth occurs.

(V) When $\tau>\tau_{\text {crit }}=\frac{\pi}{2}$ how can tumor volume exhibit runaway growth? When $\tau$ increases past the critical value $\tau_{c r i t}=\frac{\pi}{2}$ the system becomes unstable since $\alpha_{0}(\tau)$ changes from negative to positive (Figure 4): if $\tau>\frac{\pi}{2}$, and either $c_{0} \neq 0$ or $d_{0} \neq 0$, then the term $c_{0} e^{\alpha_{0}(\tau) t} \cos \left(\beta_{0}(\tau) t\right)+d_{0} e^{\alpha_{0}(\tau) t} \sin \left(\beta_{0}(\tau) t\right)$ causes $G(t)$ and $N(t)$ to undergo oscillations whose amplitudes increase without bound as $t \rightarrow \infty$. Thus, a 

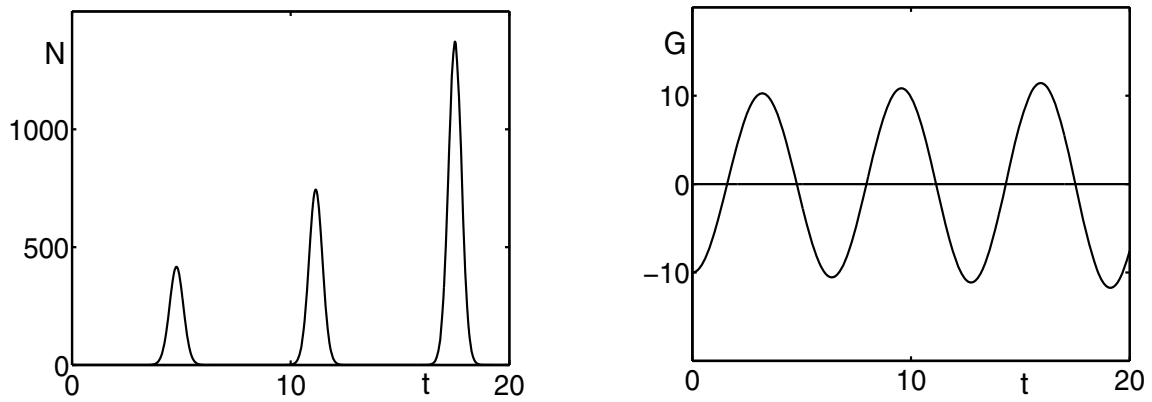

FIG. 8. Runaway growth. $N(t)$ and $G(t)$ are computed from (4.11). Left: Tumor volume $N(t)$ oscillates with amplitudes that increase sharply as $t$ increases. Right: The amplitudes of the $G(t)$ oscillations increase relatively slowly since their rate of increase is determined by $\alpha_{0}(1.6)=.084$, which is small compared to the rate of oscillation $\beta_{0}(1.6)=.987$

dormant state cannot be maintained if $\tau>\frac{\pi}{2}$, and either $c_{0} \neq 0$ or $d_{0} \neq 0$. Figure 8 illustrates this phenomenon when $\tau=1.6>\frac{\pi}{2}$. We solve

$$
\begin{aligned}
& \frac{d N(t)}{d t}=K_{1} N(t) G(t), N(0)=N_{0}>0, \\
& G(t)=c_{0} \exp \left(\alpha_{0}(1.6) t\right) \cos \left(\beta_{0}(1.6) t\right),
\end{aligned}
$$

where $K_{1}=1, \tau=1.6, c_{0}=-11, \alpha_{0}(1.6)=.084, \beta_{0}(1.6)=.987$. The left panel in Figure 8 shows that $N(t)$ oscillates with dramatically increasing amplitudes as $t$ increases. It is interesting to note that the amplitudes of the $G(t)$ oscillations increase relatively slowly since $\alpha_{0}(1.6)=.084$ is small compared to the rate of oscillation $\beta_{0}(1.6)=.987$ Similar destabilizations occur as $\tau$ increases further and passes through the higher critical values $\tau_{j}=\frac{(4 j+1) \pi}{2}, j \geq 1$. This suggests that dormancy becomes increasingly difficult to achieve at higher $\tau$ values.

5. Replication of clinical data. In this section we demonstrate how our generalized Gompertzian model (1.4) can be used to closely replicate clinical data. For many types of cancer such as carcinoma of the breast, there is a scarcity of data available that can be used to describe immune system response of untreated patients to the presence of a tumor. This is because such tumors can grow quickly and may metastasize so that the standard treatment is to begin treatment soon after tumor detection on just one mammogram. However, an important study by Heuser et al. 22 provides an excellent set of data describing growth of tumors detected on two successive mammograms for 32 untreated patients. Table 1 in 22 gives the date of each mammogram, and sizes $\left(\mathrm{mm}^{2}\right)$ of the detected tumors. Subsequently, Speer et al. 41] graphed the data for each of the 32 patients by first estimating the numbers $N_{1}$ and $N_{2}$ (the numbers of cells on successive mammograms) on the measured tumor sizes, then plotting the $N_{i}(i=1,2)$ on a $\log$ scale over time as measured in days from the initial diagnosis. For convenience they set the time of each patient's first mammogram to be $t_{1}=0$, and linearly interpolated 


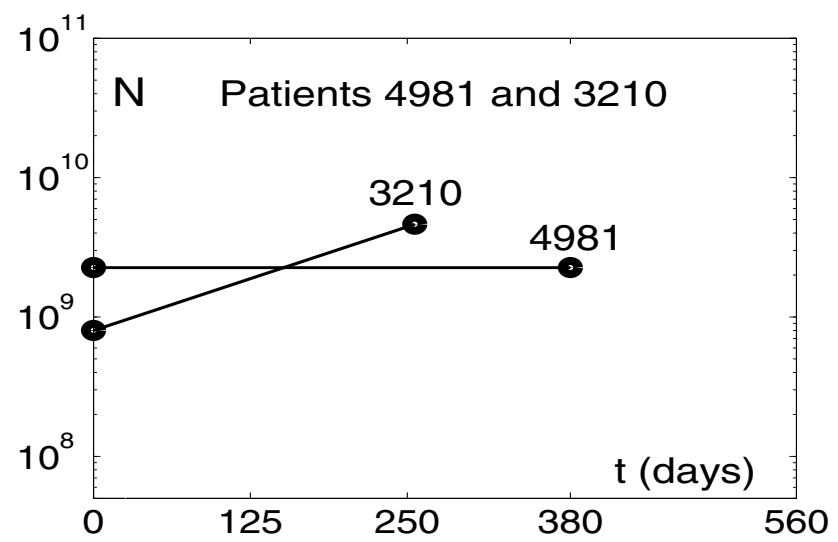

FIG. 9. Replication of two line segment graphs in Chart 5 in 41]. Line segments join two data points (large black dots) for patients 3210 and 4981 taken from Table 1 in 22. Horizontal axis denotes time (days), vertical axis denotes tumor volume (number of cancer cells). See text for details.

between the points, $\left(0, N_{1}\right)$ and $\left(t_{2}, N_{2}\right)$. Chart 5 in [41] illustrates these line segments and shows two distinct behaviors: no growth and uninhibited growth. Figure 9 shows these behaviors for two representative cases, patients 4981 and 3210 . We give the details of these data sets below and how they are replicated by our model.

Patient 4981. The first mammogram, taken $7 / 15 / 74$ shows a $20 \times 12 \mathrm{~mm}^{2}$ tumor [22] containing $2.26 \times 10^{9}$ cells [41] (Figure 9). The second, 380 days later, shows that the tumor is the same size. This "zero growth" pattern is shared by nine of the data sets. Five of these contain less than $10^{9}$ cells, hence they are classified as clinically small (as opposed to biologically small). These five data sets violate the widely acclaimed "clinical experience" (41] p. 4125) that small tumors grow rapidly.

Patient 3210. The first mammogram, taken on $5 / 25 / 75$ shows a $12 \times 11 \mathrm{~mm}^{2}$ tumor 22 . which contains $8 \times 10^{8}$ cells [41] (Figure 9). The second, 256 days later, shows a $30 \times$ $14 \mathrm{~mm}^{2}$ tumor containing $4.6 \times 10^{9}$ cells. The slope of the line segment joining $\left(0,8 \times 10^{8}\right)$ and $\left(256,4.6 \times 10^{9}\right)$, and the relative change of tumor volume, are given by

$$
\frac{\Delta N}{\Delta t}=1.5 \times 10^{7} \text { and } \frac{\Delta N}{N(0)}=4.75
$$

It follows from (5.1) that this tumor exhibits a 475 percent increase in size, i.e., an increase of 3.8 billion cells, over 256 days. This classical Gompertzian type uninhibited growth pattern is shared by over half of the 32 data sets in Table 1 [22] (and Chart 5 [41]).

Modeling goals: (I) we show (Figures 10 and 11) how our Gompertzian model (1.4) replicates tumor growth behavior shown in Figure 9 for patients 4981 and 3210; (II) For each patient we establish a baseline of prediction of plausible tumor volume evolution following the second mammogram.

(I) Replication of tumor data for patient 4981. We assume that the internal kinetics of the host play an important role in keeping tumor volume the same size when 
the two mammograms were taken. Although there is no data describing the tumor volume between mammograms, we assume that its size remains essentially constant, with at most small oscillatory fluctuations in growth or regression. To model these properties, we assume that when $0 \leq t \leq 380$, tumor volume is approximated by a periodic solution with small amplitude fluctuations centered at $N_{0}=2.26 \times 10^{6}$ cells. It follows from (2.8) that the first $\tau>0$ where periodic solutions exist is $\tau=\frac{\pi}{2 K_{2}}$, and the corresponding first eigenvalue is $\lambda_{0}=K_{2}$. It is important to note that $\tau=\frac{\pi}{2 K_{2}}$ is the largest $\tau$ value where all eigenvalues of the $G$ equation in (1.4) have nonpositive real parts, hence solutions of the $N$ equation remain bounded and the system is "stable". When $\tau=\frac{\pi}{2 K_{2}}$ the leading terms in expansion (1.12) give $G=c_{1} \cos \left(K_{2} t\right)+d_{1} \sin \left(K_{2} t\right)$. The corresponding formula for tumor volume is $N=N_{0} \exp \left(K_{1} \int_{0}^{t} G\left(t^{\prime}\right) d t^{\prime}\right)$. That is,

$$
N=N_{0} \exp \left(\frac{c_{1} K_{1}}{K_{2}} \sin \left(K_{2} t\right)+\frac{d_{1} K_{1}}{K_{2}}\left(1-\cos \left(K_{2} t\right)\right)\right) .
$$

We claim that $\tau=190$, and that boundary conditions $N(0)=N(380)=2.26 \times 10^{9}$ hold when $N_{0}$ and $K_{2}$ are given by

$$
N_{0}=2.26 \times 10^{9} \text { and } K_{2}=\frac{\pi}{380} .
$$

It follows from (5.3) that $\tau=\frac{\pi}{2 K_{2}}=190$. Substituting (5.3) into (5.2) gives

$$
N=2.26 \times 10^{9} \exp \left(\frac{380 c_{1} K_{1}}{\pi} \sin \left(\frac{\pi}{380} t\right)+\frac{380 d_{1} K_{1}}{\pi}\left(1-\cos \left(\frac{\pi}{380} t\right)\right)\right) .
$$

It follows from (5.4) that the requirement $N(0)=N(380)$ implies that $d_{1}=0$. Finally, to insure small amplitude oscillations centered at $N_{0}=2.26 \times 10^{9}$, we set $c_{1} K_{1}=\frac{\pi}{7600}$, and (5.4) becomes

$$
N=2.26 \times 10^{9} \exp \left(\frac{1}{20} \sin \left(\frac{\pi t}{380}\right)\right), 0 \leq t \leq 380 .
$$

Figure 10 gives the graph (dark circles in right panel) of (5.5): left and right panels show that (5.5) closely replicates tumor growth data for patient 4981 .

(II) Predictions of tumor volume after the second mammogram. We show how our model (1.4) can be used to give a baseline of tumor volume evolution over the six month period following the second mammogram. We focus on three diverse possibilities: (A) the tumor exhibits uninhibited growth, (B) the tumor remains the same size, (C) the tumor regresses.

CASE A. We assume that the host loses its ability to control tumor size, that uninhibited growth is initiated at $t=380$ (days), and that this growth rate is the same rate derived in (5.1) for tumor growth data for patient 3210. Thus, over a 6 month period our data based prediction suggests that tumor volume could increase in size by 2.7 billion cells. Thus, we set

$$
N(560)=2.26 \times 10^{9}+\left(1.5 \times 10^{7}\right) * 180=4.96 \times 10^{9}(\text { cells }),
$$

and the boundary conditions for $N(t)$ are

$$
N(380)=2.26 \times 10^{9} \text { and } N(560)=4.96 \times 10^{9} .
$$



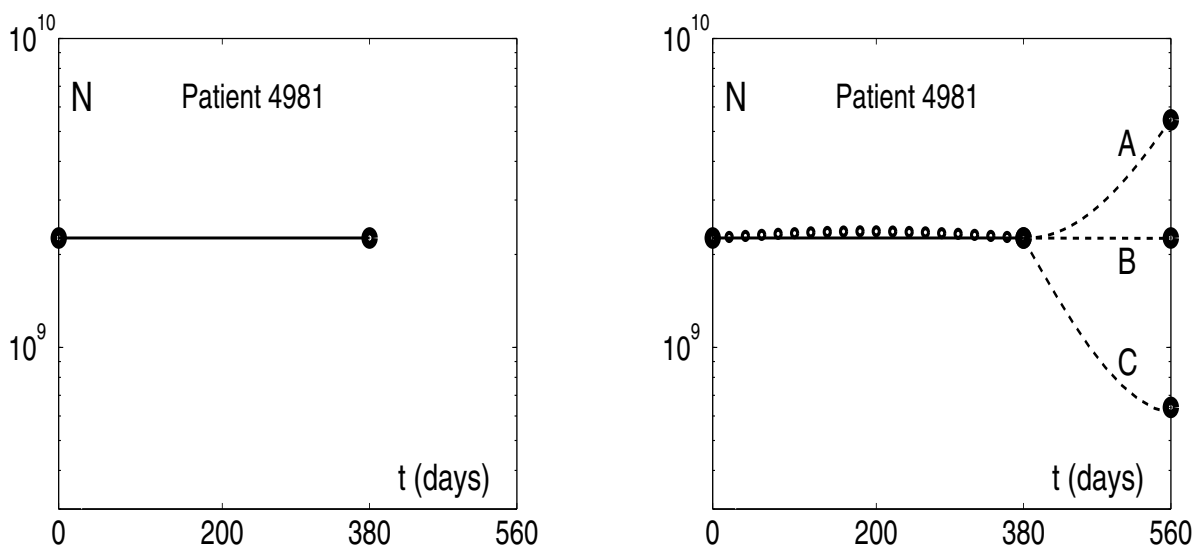

FIG. 10. Patient 4981. Left: Line segment joining data points (black dots). Vertical axis is tumor volume, horizontal axis is time since first mammogram. Right: Approximation to data (black circles) when $0 \leq t \leq 380$ and predictions (dashed curves $\mathbf{A}, \mathbf{B}, \mathbf{C}$ ) of tumor volume during the six months following the second mammogram. See text for details.

Next, we determine an appropriate range of $\tau$. Because we assume that rapid growth is initiated at $t=380$, it is reasonable to assume that $\tau$ increases past the critical value $\frac{\pi}{2 K_{2}}=190$ into the regime where $\alpha_{0}(\tau)$, the real part of the leading eigenvalue, becomes positive. At such $\tau$ values the system destabilizes and rapid growth is possible. Thus, we assume a moderate increase of $\tau$ from 190 to 195 days at $t=380$. In addition, it is natural to expect the onset of uninhibited growth at $t=380$ to exhibit classical Gompertzian growth properties. For this the appropriate leading term in expansion (4.1) is $G(t)=d_{1} \exp \left(\alpha_{0}(195) t\right) \sin \left(\beta_{0}(195) t\right)$, where $\alpha_{0}(195)$ and $\beta_{0}(195)$ are the real and imaginary parts of the leading eigenvalue $\lambda_{0}=\alpha_{0}(195)+i \beta_{0}(195)$. The corresponding tumor volume formula is

$$
N(t)=2.26 \times 10^{9} \exp \left(d_{1} K_{1} \int_{380}^{t} \exp \left(\alpha_{0}(195) t^{\prime}\right) \sin \left(\beta_{0}(195) t^{\prime}\right) d t^{\prime}\right),
$$

where $380 \leq t \leq 560$. We need to determine values for $\alpha_{0}(195), \beta_{0}(195)$ and the product $d_{1} K_{1}$. In Theorem 3.1 we proved that the eigenvalue $\lambda_{0}=\alpha_{0}(\tau)+i \beta_{0}(\tau)$ is a $C^{1}$ function of $\tau$, and that $\alpha_{0}(\tau)$ and $\beta_{0}(\tau)$ are determined by solving ODE system (3.15) with intial conditions $\left(\alpha_{0}\left(\frac{\pi}{2 K_{2}}\right), \beta_{0}\left(\frac{\pi}{2 K_{2}}\right)\right)=\left(0, K_{2}\right)$, where $K_{2}=\frac{\pi}{380}$. Thus, we solve (3.15) with $\left(\alpha_{0}(190), \beta_{0}(190)\right)=\left(0, \frac{\pi}{380}\right)$, and obtain

$$
\alpha_{0}(195)=.001 \text { and } \beta_{0}(195)=.0082 .
$$

We substitute (5.9) into (5.8) and find that boundary conditions (5.7) hold when we set $d_{1} K_{1}=-.0045$ The resultant form of $N(t)$ (dashed curve A in Figure 10, right panel) exhibits the classical Gompertzian growth characteristics predicted above.

CASE B. We assume that internal kinetics of the host continue to keep tumor volume at a constant level when $380 \leq t \leq 560$. To model this setting we assume that there is 

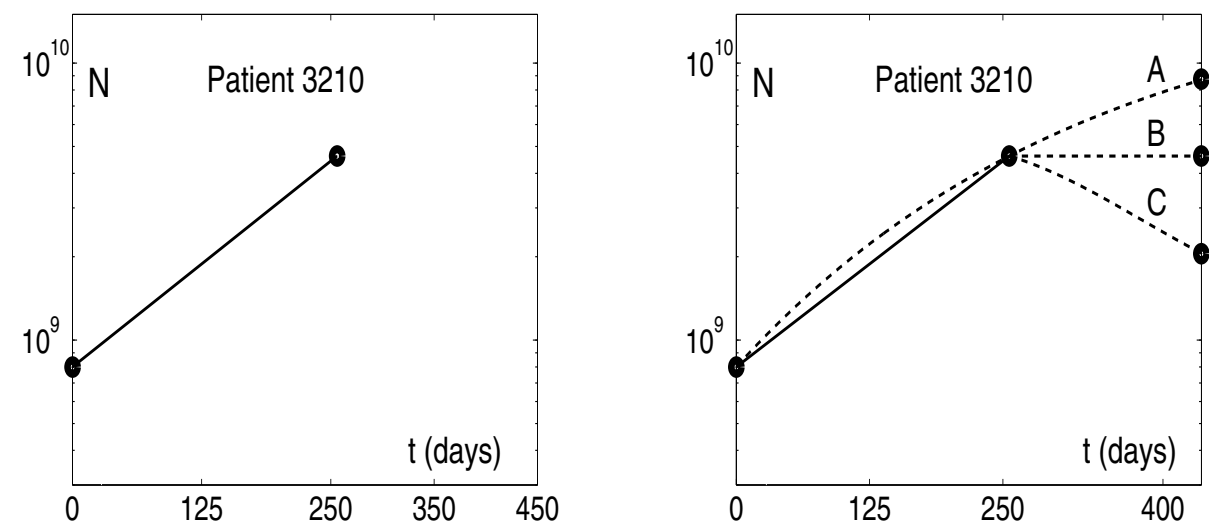

FIG. 11. Patient 3210. Left: Line segment joining data points (black dots). Right: Approximation to data (dashed curve segment) when $0 \leq t \leq 380$ and predictions (dashed curves $\mathbf{A}, \mathbf{B}, \mathbf{C}$ ) of tumor volume during the six months following the second mammogram. See text for details.

no change in $\tau$, i.e., $\tau=190$, and that formula (5.5) continues to model tumor volume when $380 \leq t \leq 560$ (dashed curve B in Figure 10, right panel).

CASE C. We assume that internal kinetics of the host cause tumor volume to regress when $380 \leq t \leq 560$. To model this setting we assume that there is no change in $\tau$, i.e. $\tau=190$, and that $c_{1} K_{1}=.0075$ in (5.5). The resultant form of $N(t)$ is indicated by dashed curve $\mathbf{C}$ in Figure 10 (right panel).

(I) Replication of tumor data for patient 3210. The boundary conditions for tumor volume (Figure 11, left) are

$$
N(0)=8 \times 10^{8} \text { and } N(256)=4.6 \times 10^{9} .
$$

Due to its rapid growth, we assume that the tumor exhibits classic Gompertzian growth characteristics when $0 \leq t \leq 256$. Thus, we set $\tau=0$ and follow Speer et al. (41], p. 4125) to obtain the classical Gompertzian approximation $N(t)=8 \times 10^{8} \exp \left(\frac{A}{K_{2}}\left(1-\exp \left(-K_{2} t\right)\right)\right)$. We find that conditions (5.10) hold when $K_{2}=.01$ and $A=.018958$. Thus (see Figure11, right),

$$
N(t)=8 \times 10^{8} \exp (1.8958(1-\exp (-.01 t))), 0 \leq t \leq 256 .
$$

A comparsion of the left and right panels of Figure 11] shows that (5.11) closely replicates tumor growth data for patient 3210 when $0 \leq t \leq 380$.

(II) Predictions of tumor volume after the second mammogram. Again, we focus on three possibilities: (A) the tumor exhibits uninhibted growth, (B) the tumor remains the same size, (C) the tumor regresses.

CASE A. When $256 \leq t \leq 436$ we assume that the tumor continues to exhibit classical Gompertzian uninhibited growth, and that $N(t)$ is again given by (5.11) (dashed curve A in Figure 11, right). 
CASE B. We assume that the tumor ceases to grow and behaves like tumor growth for patient 4981, remaining at a constant level over the 180 day interval $256 \leq t \leq 436$. Guided by our analysis of $N(t)$ for patient 4981, we set $\tau=190$ and $K_{2}=\frac{\pi}{180}$, and follow the derivation of (5.5) to obtain

$$
N(t)=4.6 \times 10^{9} \exp \left(c_{1} K_{1} \int_{256}^{t} \cos \left(\frac{\pi}{380} t^{\prime}\right) d t^{\prime}\right), 256 \leq t \leq 480 .
$$

As above, we set $c_{1} K_{1}=\frac{\pi}{7600}$ in (5.12). The resulting tumor volume formula is represented by dashed curve B in Figure 11 (right).

CASE C. Here, we assume that the tumor regresses when $256 \leq t \leq 436$. Again, we are guided by our analysis of tumor growth for patient 4981, and set $c_{1} K_{1}=\frac{15 \pi}{7600}$ in (5.12). The resulting tumor volume formula is represented by dashed curve $\mathbf{C}$ in Figure 11 (right).

Future modeling and clinical studies. It is important to develop techniques to predict which percentage of tumors evolve according to (A), (B), (C) described above for patients 4981 and 3210 . For this, we need more extensive data provided by large prospective multi-year trials. One class of tumors which falls within the framework of our generalized Gompertzian model, and for which such a long-term trial is planned, is that involving small tumors diagnosed as DCIS (ductal carcinoma in situ). This trial, called the Low Risk Ductal Carcinoma in Situ (LORIS) Trial, will monitor the evolution of DCIS tumors in 932 patients for five years, with lifelong follow up also planned. It is hoped that the results of this study, and others, will provide sufficient new data to allow our model to be modified to provide accurate predictions of long-term evolution of such tumors.

6. Conclusions. In this paper we examined the dynamic behavior of the generalized Gompertz system

$$
\begin{aligned}
& \frac{d N(t)}{d t}=K_{1} N(t) G(t), \\
& \frac{d G(t)}{d t}=-K_{2} G(t-\tau),
\end{aligned}
$$

of delay-differential equations which model host-tumor interactions. The dependent variables are tumor volume $(\mathrm{N})$, and an inhibitory variable $(\mathrm{G})$ which represents the response of the host to the tumor. Our main modeling advance is to assume that there is a delay, $\tau \geq 0$, in response of the host to the presence of the tumor. A long standing belief is that Gompertzian kinetics cannot exhibit such complicated behavior as tumor dormancy or regression [3, 41]. Indeed, Retsky et al. 38] warn us "against the attempt to imagine that tumors conform to simple rules." We agree with this principle. However, we have demonstrated that the simple, yet general Gompertzian system (6.1) can produce tumor growth scenarios which are arbitrarily complex or "irregular", and which mirror many realistic settings in the study of host-tumor interactions inherent in the evolution of tumors. We have shown that solutions of (6.1) exhibit a rich diversity of behavior, including tumor persistence, tumor regression, tumor dormancy and tumor regrowth. The first step in our approach is to develop an ODE technique to prove the existence 
and qualitative properties of infinitely many branches of solutions of the eigenvalue equation (1.6) associated with the $G(t)$ delay ODE (Theorem 3.1). We showed that for each $\tau>0$ there are infinitely many eigenvalues, and that these generate a solution of the $G(t)$ delay equation of the form

$$
\begin{aligned}
& G(t)=C_{1} e^{r_{1}(\tau) t}+C_{2} e^{r_{2}(\tau) t} \\
& +\sum_{j \in I_{1}} c_{j} \exp \left(\alpha_{j}(\tau) t\right) \cos \left(\beta_{j}(\tau) t\right)+\sum_{k \in I_{2}} d_{k} \exp \left(\alpha_{k}(\tau) t\right) \sin \left(\beta_{k}(\tau) t\right),
\end{aligned}
$$

where $e^{r_{1}(\tau) t}, e^{r_{2}(\tau) t}, \exp \left(\alpha_{j}(\tau) t\right) \cos \left(\beta_{j}(\tau) t\right)$ and $\exp \left(\alpha_{k}(\tau) t\right) \sin \left(\beta_{k}(\tau) t\right)$ are eigenfunctions.

Theorem 3.1 gives the first complete analysis of the $G(t)$ delay ODE. Using the results of Theorem 3.1, we analyzed the full system and showed the following:

(i) When $\tau>0$ is small, the host system responds, but the response is insufficient to effectively suppress tumor growth, and classical Gompertzian growth occurs.

(ii) When $\tau$ is too large, i.e., $\tau>\frac{\pi}{2 K_{2}}$, instabilities in the system can cause runaway growth of tumor volume (Figure 8). Thus, when $\tau>\frac{\pi}{2 K_{2}}$, it is unlikely that an effective response of the host can be evoked.

(iii) When $\tau$ is in an intermediate "optimal immunological response range", i.e., $\tau \in$ $\left(\tau^{*}, \tau_{\text {crit }}\right]=\left(\frac{1}{e K_{2}}, \frac{\pi}{2 K_{2}}\right]$, the response of the host may be sufficiently strong to cause tumor regression or dormancy. It is natural to expect that such an optimal response range will vary from individual to individual.

(iv) In Section 5 we demonstrated the utility of our model for replicating clinical data by showing that it accurately replicates tumor growth on successive mammograms for 32 breast cancer patients.

Extensions for future research. A fundamental difference between our approach and that of Norton and Simon [33 35] is that the Norton-Simon studies examine the effects of external input and do not address internal effects due to host-tumor interactions. A natural extension is to combine our techniques with the Norton-Simon approach by including external input in our generalized Gompertz model. This combined approach will allow us to investigate growth inhibiting effects of both external treatment and internal host-immune response. Thus, a first step is to extend system (6.1) to

$$
\frac{d N(t)}{d t}=K_{1} N(t) G(t)(1-L(t)), \frac{d G(t)}{d t}=-K_{2} G(t-\tau),
$$

where, as in 33 35], the term $L(t)$ denotes external therapy. These combined approaches may facilitate broader understanding of the complex tumor-host interactions present in the biology of cancer and may lead to new possibilities for the further development of the sequencing of cancer treatments and interventions.

Acknowledgments. The research of W. C. T. was supported by NSF Grant DMS0412370. The research of S. J. A. was supported by NIH Grants U10 CA069974 and U10 CA69651. 


\section{REFERENCES}

[1] Kenneth L. Cooke, Differential-difference equations, Internat. Sympos. Nonlinear Differential Equations and Nonlinear Mechanics, Academic Press, New York, 1963, pp. 155-171. MR0146481 (26 \#4003)

[2] M. L. Ciron, D. A. Barry, C. Cirrincione, C. Hudis, E. P. Winer, W. J, Gradishar, N. E. Davidson, S. Martino, R. Livingston, J. N. Ingle, E. A. Perez, J. Carpenter, D. Hurd, J. F. Holland, B. L. Smith, C. I. Sartor, E. H. Leung, J. Abrams, R. L. Schilsky, H. B. Muss \& L. Norton, Randomized trial of dosedense versus conventionally scheduled and sequential versus concurrent combination chemotherapy as postoperative adjuvant treatment of node-positive primary breast cancer: first report of Intergroup Trial C9741/Cancer and Leukemia Group B Trial 9741. J. Clin. Oncology 21 (2003), pp. 1431-1439.

[3] R. Demichelli, Growth of testicular-neoplasm lung metastases: tumor specific relation between two Gompertzian parameters, Eur. J. Cancer 16 (1980), pp. 1603-1608.

[4] R. Demichelli, M.W. Retsky, W.J.M. Hrushesky, M. Baum and I.D. Gukas, The effect of surgery on tumor growth: a century of investigations, Annals of Oncology 19 (2008), pp. 1821-1828.

[5] R. Demichelli, M.W. Retsky, D. E. Swartzendruber and G. Bonadonna, Proposal for a new model of breast cancer metastatic development, Annals of Oncology 8 (1997), pp. 1075-1080.

[6] Odo Diekmann, Stephan A. van Gils, Sjoerd M. Verduyn Lunel, and Hans-Otto Walther, Delay equations: Functional, complex, and nonlinear analysis, Applied Mathematical Sciences, vol. 110, Springer-Verlag, New York, 1995. MR1345150 (97a:34001)

[7] Alberto d'Onofrio, A general framework for modeling tumor-immune system competition and immunotherapy: mathematical analysis and biomedical inferences, Phys. D 208 (2005), no. 3-4, 220235, DOI 10.1016/j.physd.2005.06.032. MR2167609 (2006f:92019)

[8] L. Esserman, Y. Shieh \& I. Thompson, Rethinking screening for breast cancer and prostate cancer, JAMA 302 (5) (2009), pp. 1685-1692.

[9] B. Fisher, Laboratory and clinical research in breast cancer-a personal adventure: the David A. Karnofsky lecture, Cancer Research 10 (1980), pp. 3863-3874.

[10] B. Fisher \& E. R. Fisher, Experimental evidence in support of the dormant tumor cell, Science 130 (1959), pp. 918-919.

[11] B. Fisher, E. Montague, C. Redmund, B. Barton, D. Borland, E. R. Fisher, M. Deutsch, G. Schwarz, R. Margolese, W. Donegan, H. Volk, C. Konvolinka, B. Gardner, I. Cohn, Jr., G. Lesnick, A. B. Cruz, W. Lawrence, T. Nealon, H. Butcher \& R. Lawton, Comparison of radical mastectomy with alternative treatments for primary breast cancer: a first report of results from a prospective randomized trial, Cancer (1977) June 39 (6 Suppl), pp. 2827-2839.

[12] B. Fisher, J. H. Jeong, S. Anderson, J. Bryant, E. R. Fisher \& N. Wolmark, Twenty-five-year followup of a randomized trial comparing total mastectomy with or without radiation in the treatment of breast cancer, The New England Journal of Medicine 347 (8) (2002), pp. 567-575.

[13] B. Fisher, M. Bauer, R. Margolese, R. Poisson, Y. Pilch, C. Redmond, E. Fisher, N. Wolmark, M. Deutsch, E. Montague, et al., Five-year results of a randomized clinical trial comparing total mastectomy and segmental mastectomy with or without radiation in the treatment of breast cancer, The New England Journal of Medicine 312 (11) (1985), pp. 665-673.

[14] B. Fisher, S. Anderson, J. Bryant, R. G. Margolese, M. Deutsch, E. R. Fisher, J. H. Jeong \& N. Wolmark, Twenty-year follow-up of a randomized trial comparing total mastectomy, lumpectomy, and lumpectomy plus irradiation for the treatment of invasive breast cancer, The New England Journal of Medicine 347 (16) (2002), pp. 1233-1241.

[15] B. Fisher \& S. Anderson, The breast cancer alternative hypothesis: is there evidence to justify replacing it? Journal of Clinical Oncology 28 (3) (2009), pp. 366-374.

[16] B. Fisher, The present status of tumor immunology, Advances in Surgery, Welch and Hardy, Editors, 5 (1971), pp. 189-254.

[17] R. I. Fischer, V. T. Devita \& B. P. Hubbard, Prolonged disease free survival in Hodgkin's disease with MOPP reinduction after first relapse, Ann. Int. Medicine 90 (1979), pp. 761-763.

[18] M. A. Gimbrone, S. B. Leapman, R. S. Cotran \& J. Folkman, Tumor dormancy in vivo by prevention of neovascularization, The Journal of Experimental Medicine 136 (1972), pp. 261-276.

[19] L. Glass \& M. C. Mackey, Oscillations and chaos in physiological control systems, Science 197 (1977), pp. 287-289.

[20] B. Gompertz, On the nature of the function expressive of the law of human mortality, and on a new mode of determining the value of life contingencies, Phil. Trans. Roy. Soc. (1825), pp. 513-585. 
[21] M. Greenblatt \& P. Shubik, Tumor angiogenesis: trans filter diffusion studies by the transparent chamber technique, J. Natl. Cancer Inst. 41 (1968), pp. 111-124.

[22] L. Heuser, J. Spratt \& H. Polk, Growth rates of primary breast cancers, Cancer 43, No. 5 (1979), pp. 1888-1894.

[23] D. J. Jeffrey, D. E. G. Hare, and Robert M. Corless, Unwinding the branches of the Lambert W function, Math. Sci. 21 (1996), no. 1, 1-7. MR1390696 (97e:33003)

[24] R. M. Kaplan and F. Porzsolt, The natural history of breast cancer, Arch. Intern. Med, 168 (21) (2008), pp. 2302-2303.

[25] T. G. Karrison, D. J. Ferguson and P. Meier, Dormancy of mammary carcinoma after mastectomy, J. Natl. Cancer Inst. 91 (1) (1999), pp. 3308-3315.

[26] D. N. Krag, S. J. Anderson, T. B Julian et al., Sentinel-lymph node resection compared with conventional axillary-lymph-node dissection in clinically node-negative patients with breast cancer: overall survival findings from the NSABP B-32 randomized phase 3 trial, The Lancet Oncology 11 (10) (2010), pp. 927-933.

[27] A. K. Laird, Dynamics of tumor growth, Br. J. of Cancer 18 (1964), pp. 490-502.

[28] A. K Laird, Dynamics of growth in tumors and normal organisms, Nat. Can. Inst. Monogr. 39 (1969), pp. 15-28.

[29] B. G. Leventhal, A. S. Levine, \& R. G. Graw, Long term second remissions in acute lymphatic leukemia, Cancer 35 (1975), pp. 1136-1140.

[30] S. S. McAlister \& R. Weinberg, Tumor-host interactions: a far reaching relationship, Journal of Clinical Oncology 28(26) (2010), pp. 4022-4028.

[31] S. Meng, D. Tripathy, E. P. Frenkel, S. Shete, E. Z. Naftalis, J. F. Huth, P. D. Beitsch, M. Leitch, S. Hoover, D. Euhus, B. Haley, L. Morrison, T. P. Fleming, D. Herlyn, L. W. Terstappen, T. Fehm, T. F. Tucker, N. Lane, J. Wang, J. W. Uhr, Circulating tumor cells in patients with breast cancer dormancy, Clinical Cancer Res. 10(24) (2004), pp. 8152-8162.

[32] L. Norton R. Simon, H. Brereton \& A. Bogden, Predicting the course of Gompertzian growth, Nature 264 (1976), pp. 542-544.

[33] L. Norton \& R. Simon, Tumor size, sensitivity to therapy and the design of treatment protocols, Cancer Treat. Rep. 61 (1976), pp. 1307-1317.

[34] L. Norton \& R. Simon, The growth curve of an experimental solid tumor following radiotherapy, J. Nat. Cancer Inst. 58 (1977), pp. 1735-1741.

[35] L. Norton \& R. Simon, The Norton-Simon hypothesis revisited, Cancer Treat. Rep. 70 (1986), pp. 163-169.

[36] Tiffany O'Callaghan, The cruellest cut, New Scientist, 214, Issue 2870 (2012), pp. 42-44.

[37] C. F. J. M. Peeters, R. M. W. de Waal, T. Wobbes \& T. J. M. Ruers, Metastatic dormancy imposed by the primary tumor: does it exist in humans? Annals of Surg. Oncol. 15 (11) (2008), pp. 33083315 .

[38] M. W. Retsky, D. E. Swartzendruber, R. H. Wardwel \& P. D. Bame, Is Gompertzian or exponential kinetics a valid description of individual human cancer growth? Medical Hypotheses 33 (1998), pp. 95-106.

[39] H. E. Skipper, F. M. Schabel, Jr. \& W. S. Wilcox, Experimental evaluation of potential anticancer agents. XIII. On the criteria and kinetics associated with 'curability' of experimental leukemia, Cancer Chemoth. Rep. 35 (1964), pp. 1-111.

[40] R. Simon \& L. Norton, The Norton-Simon hypothesis: designing more effective and less toxic chemotherapuetic regimens, Nature Clinical Practice Oncology 3 (2006), pp. 406-407.

[41] J. F. Speer, V. E. Petrovsky, M. W. Retsky \& R. H. Wardwell, A stochastic numerical model of breast cancer growth that simulates clinical data, Cancer Research 44 (1984), pp. 4124-4130.

[42] W. C. Sumner \& A. G. Foraker, Spontaneous regression of human melanoma: clinical and experimental studies, Cancer 13 (1960) 79-81.

[43] P. W. Sullivan \& S. E. Salmon, Kinetics of tumor growth and regression in IgG multiple myeloma, J. Clin. Inv. 51 (1972), pp. 1607-1708.

[44] U. Veronesi, R. Saccozzi, M. Del Vecchio et al., Comparing radical mastectomy with quadrantectomy, auxiliary dissection and radiotherapy in patients with small cancers of the breast. New England Journal of Medicine 305 (1981), pp. 6-11.

[45] U. Veronesi, N. Casctinelli, L. Mariani et al., Twenty year follow-up of a randomized study comparing breast-conserving surgery with radical (Halstead) mastectomy for early breast cancer, New England Journal of Medicine 347 (1981), pp. 1227-1232. 
[46] P. H. Zahl, J. Møhlen, H. G. Welch, The natural history of invasive breast cancers detected by screening mammography, Arch. Intern Med, 168 (21) (2008), pp. 2311-2316. 